\title{
Bewertung und Klassifizierung von Bahnhaltepunkten in Nordrhein-Westfalen. Ein methodischer Ansatz zur Operationalisierung von „Transit-Oriented Development"
}

\author{
Sebastian Eichhorn (1) - Christian Gerten (1) - Christian Diller \\ Eingegangen: 9. Juli 2020 - Angenommen: 22. Dezember 2020 - Online veröffentlicht: 8. Februar 2021
}

\begin{abstract}
Zusammenfassung
Mit dem Konzept des Transit-Oriented Development (TOD) wird das Ziel verfolgt, neue Wohn- und Gewerbenutzungen an regional integrierten Standorten zu entwickeln, die den zukünftigen Bewohnerinnen und Bewohnern sowie den Beschäftigten eine gute lokale Infrastrukturausstattung, abwechslungsreiche öffentliche Räume sowie attraktive Mobilitätsalternativen zur Nutzung des eigenen Kraftfahrzeugs anbieten. In diesem Beitrag wird eine zur Operationalisierung von Transit-Oriented Development entwickelte Methodik vorgestellt und zur Bewertung und Klassifizierung von 747 nordrhein-westfälischen Bahnhaltepunkten angewandt. Mithilfe der operationalisierten TOD-Dimensionen können für Nordrhein-Westfalen fünf Cluster gebildet werden. Die Ergebnisse zeigen, dass sich die Cluster mit überdurchschnittlich hohen TOD-Werten in den Dimensionen Destination Accessibility, Density und Diversity vorwiegend im Rhein-Ruhr-Raum sowie in den solitären Städten des Landes finden lassen. In den peripheren Räumen treten hauptsächlich die Cluster mit geringeren TOD-Werten auf. Die Ergebnisse weisen darauf hin,
\end{abstract}

$\triangle$ Sebastian Eichhorn, ILS - Institut für Landes- und Stadtentwicklungsforschung, Brüderweg 22-24, 44135 Dortmund, Deutschland

sebastian.eichhorn@ils-forschung.de

$\triangle$ Christian Gerten, ILS - Institut für Landes- und Stadtentwicklungsforschung, Brüderweg 22-24, 44135 Dortmund, Deutschland

christian.gerten@ils-forschung.de

$\triangle$ Prof. Dr. Christian Diller, Institut für Geographie, Bereich Raumplanung und Stadtgeographie, Justus-Liebig-Universität Gießen, Senckenbergstraße 1, 35390 Gießen, Deutschland Christian.Diller@geogr.uni-giessen.de

(c) (1) (2) (ㅇ) 2021 Eichhorn; licensee oekom verlag. This Open Access article is published under the Creative Commons Attribution-ShareAlike 4.0 International Licence. dass es eine Diskrepanz zwischen der Verfügbarkeit von potenziell entwickelbaren Flächen und dem TOD-Grad gibt. Im Umfeld der Haltepunkte der hochleistungsfähigen Bahnstrecken in Regionen mit hoher Wohnungsnachfrage stehen kaum Flächenpotenziale zur Verfügung. Große Flächenpotenziale gibt es hingegen an den Haltepunkten mit geringen TOD-Werten und einem geringeren Druck auf dem Wohnungsmarkt. Die Cluster ermöglichen es, spezifische Handlungsempfehlungen für die Stadtplanung und politische Entscheidungen zu formulieren, um eine erfolgreiche Implementation von TransitOriented Development zu unterstützen.

Schlüsselwörter: Transit-oriented development Operationalisierung - Klassifizierung - Siedlungsentwicklung Verkehrsentwicklung - Nordrhein-Westfalen

Evaluating and classifying railway stations in North Rhine-Westphalia. A methodological approach to operationalise Transit-Oriented Development

\section{Abstract}

The concept of Transit-Oriented Development (TOD) aims to develop new residential and commercial areas in regionally integrated sites that provide future residents and employees with good local infrastructure, diversified public spaces and attractive mobility alternatives so that they do not have to use their own car. This paper presents a methodology for the operationalisation of Transit-Oriented Development and applies it to evaluate and cluster of 747 railway stations in North Rhine-Westphalia, Germany. Using the operationalised TOD dimensions, five interpretable clusters can be defined for North Rhine-Westphalia. The results show that the clusters 
with above-average TOD values in the dimensions destination accessibility, density and diversity can be found mainly in the Rhine-Ruhr region and in the solitary cities of North Rhine-Westphalia. In the peripheral areas, primarily clusters with lower TOD values occur. The findings indicate that there is a discrepancy between the availability of potentially developable building land and the degree of Transit-Oriented Development. In the surroundings of the railway stations of the high-capacity railway lines in regions with high demand for housing, there is barely any potential building land available. The majority of potential building land is located at railway stations with below-average TOD values and less pressure on the housing market. The clusters make it possible to formulate specific recommendations for action for urban planners and political decision makers in order to support a successful implementation of Transit-Oriented Development.

Keywords: Transit-oriented development Operationalisation = Classification - Settlement development - Transport development - North RhineWestphalia

\section{Einleitung}

Die Siedlungsentwicklung im Außenbereich von Städten und Gemeinden gehört zu den wesentlichen Treibern einer nach wie vor hohen Flächenneuinanspruchnahme in Deutschland (vgl. Behnisch/Kretschmer/Meinel 2018). Dieser Trend wird dabei sowohl von Prozessen der Reurbanisierung, also des relativen Bedeutungsgewinns der Kernstädte, als auch vom Wachstum des engeren oder erweiterten Umlandes der Kernstädte getrieben (Osterhage 2018).

Zum einen führt Reurbanisierung in vielen Städten zu angespannten Immobilienmärkten mit steigenden Boden- und Mietpreisen. Die Schaffung von bezahlbarem Wohnraum wird dadurch schwerer, da notwendige Entwicklungsflächen fehlen. Das Wohnen in den Kernstädten ist somit häufig nur noch für Spitzenverdiener möglich und Menschen mit geringerem Einkommen werden in das (auch weiter entfernte) Umland abgedrängt. Zum anderen ist die Nachfrage nach Bauland für Ein- und Zweifamilienhäuser im Grünen weiterhin hoch (Dechent/Wagner 2020: 49 ff.). Selbst dort, wo in den Innenstädten noch ausreichend Wohnraum vorhanden wäre, reagieren Kommunen mit der Ausweisung von neuem Bauland, um dieser Nachfrage nachzukommen.

Beide Faktoren begünstigen eine Verlagerung der Siedlungsentwicklung in die Außenbereiche und eine voranschreitende Zersiedelung der Landschaft. Wenn dabei zudem monofunktionale Siedlungsstrukturen (z. B. Ein- und Zweifamilienhausgebiete) abseits bestehender Zentren entstehen, führt dies zu einer weiteren strukturellen Pkw-Ab- hängigkeit des suburbanen Raums, die sich in einem erhöhten Verkehrsaufkommen niederschlägt (Beckmann/Dosch 2018: $21 \mathrm{f}$.). Zunehmende räumlich-funktionale Verflechtungen tragen bei zu höheren $\mathrm{CO}^{2}$-Emmissionen des Verkehrs, dem Verlust von Biodiversität und natürlichen Lebensräumen sowie der Störung des Landschaftsbildes und höheren Infrastrukturkosten (Fina 2018: $111 \mathrm{f}$.).

Transit-Oriented Development (TOD) ist ein Konzept, das diesen negativen Effekten entgegenwirken soll, indem es die Siedlungs- und Verkehrsentwicklung integriert betrachtet und Haltepunkte des öffentlichen Verkehrs als wichtige räumliche Orientierungspunkte für eine nachhaltige Stadt- und Regionalentwicklung nutzt. Während sich im internationalen Diskurs der Begriff des TransitOriented Development etabliert hat, werden in Deutschland ähnliche Konzepte bereits seit den 1960er-Jahren unter den Stichworten „Punkt-axiale Siedlungsentwicklung“ oder „Siedlungsachsen“ verfolgt (Münter/Osterhage 2018: 1187f.). Mit Fragen nach Erreichbarkeit, Fußgängerfreundlichkeit, Nutzungsmischung und Dichtewerten geht Transit-Oriented Development dabei über das bloße Bauen von Wohnhäusern und Parkplätzen für Berufspendlerinnen und -pendler in der Nähe eines Schienenanschlusses hinaus. TOD-Projekte zielen auf eine enge Verknüpfung von verdichtetem Wohnen und Arbeiten mit einer guten lokalen Infrastrukturausstattung, attraktiven öffentlichen Räumen und einer weit gefächerten Auswahl an Mobilitätsoptionen ab (Jamme/Rodriguez/Bahl et al. 2019).

Der vorliegende Beitrag knüpft an diesen internationalen Diskurs an und verfolgt insgesamt drei Ziele. Erstens steht die Entwicklung eines methodischen Ansatzes im Fokus, mit dem möglichst viele der in der Literatur diskutierten TOD-Dimensionen operationalisiert werden können. Zweitens wird am Beispiel des Bundeslandes NordrheinWestfalen die Anwendung der entwickelten Methodik getestet. Dabei werden 747 Haltepunkte des schienengebundenen Regionalverkehrs bewertet und mithilfe einer hierarchischen Clusteranalyse klassifiziert. Drittens werden auf der Grundlage der Ergebnisse mögliche Empfehlungen für Stadtplaner/-innen und politische Entscheidungsträger/-innen zur Stärkung einer integrierten Siedlungs- und Verkehrsentwicklung diskutiert.

Im zweiten Kapitel dieses Beitrags wird zunächst der internationale Stand der Forschung zu diesem Thema aufbereitet. Hierzu werden die Grundidee sowie die unterschiedlichen Forschungsausrichtungen des Transit-Oriented Development erläutert. Den Kern des Beitrags bildet eine empirische, GIS-basierte TOD-Analyse, die in Kapitel 3 dargelegt wird. Nach der Darstellung der Ergebnisse in Kapitel 4 schließt der Beitrag mit einer Abschätzung darüber, wie die Ergebnisse für eine integrierte Siedlungs- und Verkehrs- 
entwicklung in der Planungspraxis genutzt werden können (Kapitel 5).

\section{Stand der internationalen Forschung zum „Transit-Oriented Development"}

\subsection{Grundidee und Forschungsausrichtungen des "Transit-Oriented Development"}

Der Begriff des Transit-Oriented Development wurde ursprünglich von Calthorpe (1993) geprägt. Die Grundidee einer engen Verknüpfung von Siedlungsentwicklung und schienengebundenem öffentlichem Nahverkehr ist jedoch deutlich älter. Bezüge finden sich bereits im Konzept der Nachbarschaftseinheit von Perry (1929) und dem von Fritz Schumacher für Hamburg entwickelten Modell einer an ÖPNV-Achsen orientierten regionalen Siedlungsentwicklung (,Federplan“) aus dem Jahr 1919 (Bose 1994). Heutzutage gelten in Europa insbesondere die Städte Stockholm und Kopenhagen als gute Beispiele für eine regionale und integrierte Stadterweiterungsplanung, die konsequent an ÖPNV-orientierten Siedlungsachsen ausgerichtet wurde. Aber auch in den USA mit einer insgesamt vergleichsweise schwach ausgeprägten regionalplanerischen Tradition wurden - zumindest von wissenschaftlicher Seite - schon seit den 1960er-Jahren Überlegungen zu Transit-Oriented Development angestellt (Meyer/Kain/Wohl 1965; Cervero 1984; National Academies of Sciences, Engineering, and Medicine 2004). In der Praxis wurden diese zunächst jedoch kaum umgesetzt (Carlton 2019). Erst als sich in den 1990er-Jahren die TOD-Forschung intensivierte, wurde auch in den USA zunehmend die Umsetzung in der Praxis erprobt. Auch auf anderen Kontinenten, vor allem in den wachstumsstarken asiatischen Metropolregionen, wurden in den letzten Jahren TOD-Überlegungen forciert.

Die Grundidee des Transit-Oriented Development ist es, die Siedlungsentwicklung an Knotenpunkten des öffentlichen Personennahverkehrs (ÖPNV) zu orientieren, wobei neben Regionalbahn- und U-Bahnlinien auch Straßenbahnen und Busse Berücksichtigung finden können (Ibraeva/ Correia/Silva et al. 2020: $110 \mathrm{ff}$.). Der Begriff des Public Transport-Oriented Development (PTOD) wäre insofern präziser, wird jedoch kaum verwendet (Hickman/Hall 2008: $323 \mathrm{ff}$.). Kennzeichnend für die meisten TOD-Analysen ist der Node-Place-Ansatz nach Papa und Bertolini (2015). Die Annahme ist, dass ein verbessertes Verkehrsangebot eines Standortes durch die Optimierung der Erreichbarkeit günstige Bedingungen für die weitere Entwicklung des Standortes schafft. Im Umkehrschluss schafft die Entwicklung eines Standortes aufgrund einer wachsenden
Verkehrsnachfrage günstige Bedingungen für die weitere Entwicklung des Verkehrssystems. TOD-Analysen beinhalten nach Papa und Bertolini (2015) demzufolge vier Hauptuntersuchungsgegenstände: 1) Die Verbindung zwischen dem Bahnnetz und der Landnutzung und dem sich daraus ergebenden TOD-Grad, 2) den Zusammenhang zwischen TOD-Grad, Siedlungsstruktur und Mobilitätsverhalten, 3) die Auswirkung der Zugänglichkeit von Haltepunkten auf das Reiseverhalten sowie 4) die Auswirkung des TODGrades auf die Siedlungsstruktur und die Zugänglichkeit der Bahnhaltepunkte. Es gibt sowohl Untersuchungen, die die Bahnstationen bestehender Bahnnetze auf ihre TODQualität bewerten, als auch Untersuchungen, die versuchen, optimale Lösungen für neue Netze zu entwickeln. Mittlerweile hat sich, wie der aktuelle und umfassende Überblick von Ibraeva, Correia, Silva et al. (2020) verdeutlicht, die TOD-Forschung ausdifferenziert. Insgesamt lassen sich drei Hauptforschungsstränge unterscheiden:

TOD-Basisstudien: In diesen meist GIS-basierten Studien werden auf der Grundlage einer Definition von TODDimensionen deskriptiv in einer Region TOD-Einzugsbereiche abgegrenzt und damit die Verbindung von Daten des Schienenverkehrs und der an Bahnhöfe angebundenen Bevölkerung, Arbeitsplätze und Nutzungen hergestellt, woraus sich für die einzelnen Bereiche der Region ein TOD-Grad ermitteln lässt. Für diese durch den ÖPNV besonders gut angebundenen Bereiche lassen sich unter anderem Flächenpotenziale für die weitere Verdichtung ermitteln oder die Bedeutung einzelner Knotenpunkte für das gesamte Verkehrsnetz bewerten. Diese Studien und ihre zugrunde liegenden TOD-Typologien sind für diese Arbeit von besonderem Interesse und werden in Kapitel 2.2 ausführlicher erläutert.

TOD-Wirkungsanalysen: Auf der Grundlage der Abgrenzung der TOD-Einzugsbereiche können komplexe Wirkungsanalysen zu Effekten der Verknüpfung von ÖPNV und Siedlung durchgeführt werden. Methodisch werden hier entweder Zeitreihenanalysen durchgeführt, in denen beispielsweise Daten vor dem Bau einer Schienenverbindung und danach miteinander verglichen werden und/oder es werden die Entwicklungsdaten von Stadtregionsteilen innerhalb der TOD-Einzugsbereiche mit jenen außerhalb dieser Bereiche verglichen. Untersucht wurden hierbei insbesondere Effekte auf die Verkehrsträgernutzung (Cervero 1995; Hickman/Hall 2008; Knowles 2012; Singh/Fard/Zuidgeest et al. 2014), die Siedlungsentwicklung, Nutzungsstruktur und städtebauliche Form (Papa/Bertolini 2015; Dong 2016; Zhao/Yang/Kong et al. 2018; Niu/Hu/Shen et al. 2019), den Bodenmarkt, Wohnungen und Arbeitsplätze (Zemp/ Stauffacher/Lang et al. 2011; Kay/Noland/DiPetrillo 2014; Renne/Tolford/Hamidi et al. 2016; Xu/Zhang/Aditjandra 2016; Loo/Cheng/Nichols 2017; Yu/Pang/Zhang 2018) so- 
wie die Lebens- und Umweltqualität (Gilat/Sussman 2003; Kahn 2007; Lin/Li 2008; Kamruzzaman/Wood/Hine et al. 2014; $\mathrm{Ma} / \mathrm{Chen} / \mathrm{Li}$ et al. 2018). Insgesamt stellt sich die Bilanz von TOD-Konzepten, wenn sie denn konsequent implementiert werden, aus planerischer Sicht bezogen auf Siedlungs- und Verkehrsentwicklung und Umweltqualität als positiv dar. Vor allem was die Frage der Veränderungen von Bevölkerungsstrukturen (Kim/Ahn/Choi et al. 2016) angeht, wird allerdings der TOD-Ansatz durchaus auch kritisch auf seine unerwünschten Nebenwirkungen, insbesondere forcierte Gentrifizierung hin, diskutiert und analysiert (Kahn 2007; Padeiro/Louro/da Costa 2019).

TOD-Planimplementationsstudien: Diese politikwissenschaftlichen Studien sind mitunter zwar mit GIS-Basis-Analysen verbunden, jedoch insgesamt eher qualitativ ausgerichtet. Hierin geht es vor allem um die Frage der Entstehung und Umsetzung von TOD-Konzepten sowie um die Hemmnisse auf den Ebenen von Politik, Verwaltung, Planung und Investoren (Bahn.Ville 2-Konsortium 2010; Greene/Mora/ Waintrub et al. 2016; Greene/Mora/Figueroa et al. 2017; Thomas/Pojani/Lenferink et al. 2018; Carlton 2019; Paulsson 2020). Ausführlicher wird nachfolgend auf die TODBasisstudien und die ihnen zugrunde liegenden TOD-Dimensionen eingegangen.

\subsection{TOD-Dimensionen und TOD-Basisstudien}

Die ersten TOD-Basisstudien waren methodisch einfache Darstellungen, um die Bezüge zwischen der Zugänglichkeit im Schienennetz und der Nutzung ausgewählter Standorte deutlich zu machen (Bertolini 1996; Bertolini 1999). Die Operationalisierung erfolgte dabei ursprünglich nur nach siedlungsstrukturellen bzw. städtebaulichen Kriterien. Die drei Betrachtungsdimensionen waren Density (Dichte), Diversity (Nutzungsmischung) und Design (Gestaltung des näheren Einzugsbereiches) (Cervero/Kockelman 1997). Beim Kriterium Density wurde zum Teil zwischen Bevölkerungsund Arbeitsplatzdichte auf der einen und Siedlungsdichte auf der anderen Seite unterschieden. Mit der Zeit wurde dieser 3-D-Forschungsrahmen jedoch sukzessive erweitert: Im sogenannten 6-D-Modell sind zu den o.g. Aspekten die verkehrsbezogenen Dimensionen Destination Accessibility (Angebotsqualität der Station im Gesamtnetz), Distance to Transit (verkehrliche Erreichbarkeit der Station im Einzugsgebiet) und Demand Management (Nachfragesteuerung) hinzugekommen (Jamme/Rodriguez/Bahl et al. 2019). Vor allem der letzte Aspekt ist allerdings bislang eher als normative Anforderung an Transit-Oriented Development zu sehen und wurde, wie Tabelle 1 zeigt, nur in wenigen Studien operationalisiert. Eine Sonderstellung nimmt die in Tabelle 1 ebenfalls aufgeführte Dimension Development Potential (entwickelbare Flächenpotenziale im
Einzugsbereich) ein, die aktuell nicht Kern des 6-D-Modells ist, aus Sicht der Autoren aber eine inhaltlich sinnvolle Ergänzung darstellen würde (vgl. Kapitel 3.2). Insgesamt kann das 6-D-Modell als normative Messlatte für TOD-Studien und -Konzepte gelten.

Methodenorientierte GIS-basierte TOD-Analysen wurden für einzelne Einzugsbereiche seit den 1990er-Jahren erstellt (Banai 1998; Banai 2005), dabei wurden die TOD-Kriterienkataloge und Berechnungsalgorithmen im Laufe der Jahre kontinuierlich erweitert (Lin/Gau 2006; Sahu 2018). Die methodischen Fortschritte werden insbesondere auch durch einen Blick in die unterschiedlichen Studien deutlich. So enthält Tabelle 1 solche Studien, die die TOD-Analysen nicht nur auf einzelne Stationsbereiche, sondern auf ganze Städte bzw. Stadtregionen angewendet haben und somit Ergebnisse ableiten konnten, die auch für die übergeordnete Gesamtplanung von Relevanz sind. Augenfällig ist, dass die Anzahl an TOD-Studien vor allem für asiatische Stadtregionen (z. B. Shanghai, Singapur, Peking, Teheran, Jiaomei) in den letzten Jahren zugenommen hat. Dies dürfte zum einen auf die hohe Entwicklungsdynamik zurückzuführen sein, die zu einem massiven Ausbau des Schienennetzes führte und eine an Nachhaltigkeit orientierte Gesamtplanung immer mehr erforderte. Zum anderen scheint die Datenlage ein weiterer Treiber für diesen Trend zu sein, die komplexe Modellierungen mit differenzierten Variablenkatalogen schon früh ermöglichte (Chen/Lin 2015: 445 ff.; Lyu/ Bertolini/Pfeffer 2016: $40 \mathrm{ff}$.).

Es ist dennoch festzuhalten, dass sich die Mehrheit der Studien auf ausgewählte Dimensionen beschränkt. Insbesondere die im Vergleich schwerer quantifizierbaren Dimensionen Demand Management und Design wurden seltener in den Analysen berücksichtigt. Die in Tabelle 1 aufgelisteten Studien lassen sich in zwei Hauptgruppen unterteilen:

Place-orientierte Studien analysieren die Bereiche entlang von Bahnlinien relativ intensiv auf ihre Bevölkerungsund Beschäftigtenentwicklung (Gilat/Sussman 2003; Hickman/Hall 2008; Greene/Mora/Figueroa et al. 2017) oder auf ihre Nutzungsstruktur und Flächenpotenziale (Boarnet/ Compin 1999; Curtis 2008; Lyu/Bertolini/Pfeffer 2016; Loo/Cheng/Nichols 2017; Niu/Hu/Shen et al. 2019; Liang/Du/Wang et al. 2020). In ihnen bildet zwar das Schienennetz den Ausgangspunkt der Analyse, die Anbindungsqualität des Netzes geht jedoch kaum in die Bewertung ein. Dafür werden in diesen Untersuchungen zum Teil die städtebaulichen Gestaltungsqualitäten der näheren Einzugsbereiche detailliert bewertet (Cervero/Kockelman 1997; Singh/Fard/Zuidgeest et al. 2014). In dem am Beispiel der australischen Gold Coast entwickelten LUPTAI-Ansatz wird zudem die Erreichbarkeit einzelner Funktionen wie Einkaufen, Gesundheit und Bildung detailliert ermittelt 
und auch das Straßennetz einbezogen (Pitot/Yigitcanlar/ Sipe et al. 2006).

Integrierte Node-Place-Studien konzentrieren sich dagegen auf den Kern des Node-Place-Ansatzes und operationalisieren sowohl die Bedeutung der Haltepunkte im regionalen Schienennetz (node) als auch die erreichte Bevölkerung bzw. die erreichten Beschäftigten sowie die Nutzungsformen im Umfeld (place). Dafür ist in diesen Ansätzen die Betrachtung des Flächen- und Verdichtungspotenzials - abgesehen von Chen und Lin (2015) - vergleichsweise weniger stark ausgeprägt. Die in diesem Sinne umfassendsten und bezogen auf den Node-Place-Ansatz ausgewogensten Studien sind jene von Lyu, Bertolini und Pfeffer (2016) für Peking, Monajem und Nosratian (2015) für Teheran und Reusser, Loukopoulos, Stauffacher et al. (2008) für die Schweiz, obwohl bei Letzteren mit geringerem Variablenumfang gearbeitet wurde. Sie berücksichtigten nicht nur die Bedeutung der Haltepunkte im regionalen Netz, sondern auch die Anbindungsqualität der Haltepunkte innerhalb ihrer Einzugsbereiche - ein Kriterium, das ansonsten nur wenige Studien einbeziehen (Bahn.Ville 2-Konsortium 2010; Higgins/Kanaroglou 2016). Zu erwähnen ist auch der Ansatz von Büttner, Kinigadner, Ji et al. (2018) für die Region München, in dem zusätzlich zu den Flächenpotenzialermittlungen die Vulnerabilitäten der einzelnen Kommunen bezogen auf die ÖPNV-Erreichbarkeit ermittelt wurden.

Eine Zwischenstellung nimmt die Studie von Strong, Ozbek, Sharma et al. (2017) für Denver ein, bei der zwar die Einbindung der Haltepunkte in das überregionale Netz nicht operationalisiert wurde, dafür aber die Anbindung der Haltepunkte im Einzugsbereich mit einer Reihe von Variablen sehr differenziert erfasst ist. Hervorzuheben ist, dass zudem die eher selten einbezogenen TOD-Dimensionen $D e$ sign und Demand Management operationalisiert wurden.

Für den deutschsprachigen Raum sind vier TOD-Analysen herauszustellen und in ihren Ergebnissen ausführlicher darzustellen: Die komplexe und zudem vergleichende Studie von Seidemann (2016) wurde für drei Regionen in Baden-Württemberg (Mittlerer Oberrhein, Rhein-Neckar und Ostwürttemberg) erarbeitet. Diese kam zu dem Ergebnis, dass Innenentwicklungspotenziale im Einzugsbereich von Bahnhöfen häufiger auftreten als in anderen Bereichen, diese aber schwierigere Voraussetzungen zur Aktivierung aufweisen. Dennoch waren in den Fallbeispielen hohe Aktivierungsraten von $6 \%$ pro Jahr zu verzeichnen. An diesen Standorten konnte - im Vergleich zu anderen Räumen ein erhöhter Modal Split zugunsten des Schienenverkehrs festgestellt werden. Innerhalb des deutsch-französischen Kooperationsprojektes „Bahnville“ wurden exemplarisch zwei Bahnstrecken in der Region Rhein-Main und der Region Rhône-Alpes einer TOD-Analyse unterzogen, womit die regionalen Entwicklungspotenziale und Möglichkeiten ihrer
Aktivierung illustriert wurden (Bahn.Ville 2-Konsortium 2010). In diesem Zusammenhang ist zudem die Untersuchung von Reusser, Loukopoulos, Stauffacher et al. (2008) interessant, als hier zum einen eine nicht nur stadtregionale, sondern polyzentrische landesweite Klassifizierung aller Bahnstationen der Schweiz vorgenommen wurde. Zum anderen zeigt die Darstellung, wie ein anfänglich formulierter umfassender Ideal-Indikatorensatz zur Ausfüllung der TOD-Dimensionen sich in der Operationalisierung deutlich ausdünnte, was die Problematik der Datenverfügbarkeit zur Messung des 6-D-Modells nochmals verdeutlicht. Zwar sind im letztlich gerechneten Modell vier TOD-Dimensionen abgedeckt, allerdings nur mit sehr rudimentären Variableninformationen. Dennoch liefert die landesweite Klassifizierung der Schweizer Bahnhöfe ein plausibles und informatives Bild. Die Untersuchung von Büttner, Kinigadner, Ji et al. (2018) zeigt für die Region München erstens sehr kleinteilig für die einzelnen Kommunen an, welche Bereiche aus der Perspektive der ÖPNV-Anbindung vergleichsweise unterversorgt sind und in welchen Bereichen unter dem Kriterium der ÖPNV-Erreichbarkeit eine Erhöhung der Bevölkerungsdichten erfolgen könnte. Zweitens wird eine kommunal differenzierte Einschätzung der verkehrlichen Vulnerabilität vorgenommen.

Als Fazit bleibt festzuhalten, dass der normative Anspruch an Transit-Oriented Development, die sechs Betrachtungsdimensionen ausgewogen auszufüllen, bislang von noch keiner empirischen Basisstudie eingehalten werden konnte, auch wenn einzelne Studien diesem Anspruch sehr nahe kommen. Auch die hier vorgelegte Analyse verfolgt das Ziel einer breiteren Abdeckung der TODDimensionen.

\section{Empirie}

\subsection{Untersuchungsraum}

Nordrhein-Westfalen ist das bevölkerungsreichste Bundesland in Deutschland und mit einer Bevölkerungsdichte von 525,7 Einwohnerinnen/Einwohnern pro $\mathrm{km}^{2}$ (Stand 2018) besonders dicht besiedelt. Mit 565.798 ha für Siedlung (17\%) und 238.539 ha für Verkehr (7\%) sind bereits heute (Stand 2018) ein Viertel der gesamten Katasterfläche baulich geprägt. Über $55 \%$ der Siedlungsfläche entfallen dabei auf Wohnbauflächen und Flächen mit gemischter Nutzung. ${ }^{1}$ Neben einem hohen Urbanisierungsgrad ist Nordrhein-Westfalen durch seine polyzentrische Siedlungs-

\footnotetext{
1 https://www.landesdatenbank.nrw.de/ldbnrw/online (07.12.2020).
} 
struktur geprägt, welche sich in der hohen Dichte an Oberund Mittelzentren, aber auch in dem sehr engmaschigen Schienennetz widerspiegelt. 2015 verfügte das Land mit über $5.500 \mathrm{~km}$ über das zweitlängste und mit $161,9 \mathrm{~km}$ pro $1.000 \mathrm{~km}^{2}$ über das dichteste Schienennetz in Deutschland. ${ }^{2}$

Die dynamische Bevölkerungsentwicklung der letzten Jahre hat in vielen nordrhein-westfälischen Städten zu angespannten Wohnungsmärkten geführt und die Wohnraumfrage erneut auf die politische Agenda gebracht. Mit einem durchschnittlichen jährlichen Bevölkerungswachstum von rund 0,8\% zwischen 2011 und 2018 nehmen hierbei die Städte Düsseldorf, Bonn, Köln, Aachen und Münster die Spitzenplätze ein. Lediglich sieben Kreise, darunter der Hochsauerlandkreis, der Märkische Kreis und Höxter, wiesen im selben Zeitraum einen durchschnittlichen jährlichen Bevölkerungsrückgang von $0,2 \%$ auf (Landesbetrieb für Information und Technik Nordrhein-Westfalen (IT.NRW) 2020). ${ }^{3}$

Nordrhein-Westfalen bietet sich als Fallstudie an, da zum einen die integrierte Siedlungs- und Verkehrsentwicklung an strategisch sinnvollen Punkten in einem stark urbanisierten und dynamisch wachsenden Raum besonders wichtig ist und zum anderen die Datenlage im Vergleich zu anderen Bundesländern eine umfangreiche und flächendeckende Operationalisierung der TOD-Dimensionen möglich macht.

\subsection{Methodik}

Ziel der Analyse ist die Bewertung und Klassifizierung von Haltepunkten des schienengebundenen Regionalverkehrs, die sich aus dem Blickwinkel des Transit-Oriented Development für eine Siedlungsentwicklung besonders gut eignen. Die Bewertung der Haltepunkte erfolgt multikriteriell und stützt sich auf das in der Literatur diskutierte 6-D-Modell (vgl. Kapitel 2.2). Die Bewertungssystematik orientiert sich an dem von Bertolini $(1996,1999)$ entwickelten Node-place-Ansatz, so dass sowohl Eigenschaften des Haltepunktes (node) als auch des Einzugsbereiches (place) einbezogen und erfasst werden. Die hier analysierten Haltepunkte werden mindestens von einem Regionalexpress, einer Regionalbahn oder einer S-Bahn angefahren, schienengebundene Haltepunkte für U- und Trambahnen werden nicht berücksichtigt. Nach dieser Abgrenzung können für Nordrhein-Westfalen 747 Haltepunkte analysiert werden. Die für diese Studie genutzten Einzugsbereiche (Isochrone)

\footnotetext{
2 https://www.destatis.de/DE/Themen/Branchen-Unternehmen/ Transport-Verkehr/Unternehmen-Infrastruktur-Fahrzeugbestand/ Tabellen/schieneninfrastruktur.htm (07.12.2020).

3 https:// www.landesdatenbank.nrw.de/ldbnrw/online (07.12.2020).
}

ergeben sich aus einer maximalen Gehzeit von 15 Minuten ausgehend vom jeweiligen Haltepunkt und wurden mithilfe des OpenTripPlanner berechnet. Im Vergleich zu Studien mit festen Radien (Greene/Mora/Figueroa et al. 2017; Loo/ Cheng/Nichols 2017; Yang/Pojani 2017; Niu/Hu/Shen et al. 2019; Liang/Du/Wang et al. 2020) bieten Isochrone - aus der Sicht der Autoren - eine realistischere Abgrenzung der fußläufig erreichbaren Einzugsbereiche.

Insgesamt werden in der vorliegenden Studie fünf der sechs ,klassischen ‘ TOD-Dimensionen operationalisiert. Lediglich die Dimension Design bleibt unberücksichtigt, da eine fußgänger- und radfahrerfreundliche Stadtgestalt (z. B. im Hinblick auf Sauberkeit, Architektur, Verkehrsbelastung) nach Ansicht der Autoren nur unzureichend über eine flächendeckende quantitative Analyse per Fernerkundung erfasst werden kann. Ergänzt werden die fünf TOD-Dimensionen um die Dimensionen Development Costs und Development Potential. Die Dimension Development Costs (Bodenpreise im Einzugsbereich) dient dazu, das 6-D-Modell um eine finanzielle Perspektive zu erweitern, indem die Kosten, die bei zukünftigen Wohnbauvorhaben in Einzugsbereichen anfallen können, berücksichtigt werden (Schmitt/ Seidl 2006: $97 \mathrm{ff}$.; Spars 2017). Eine entsprechende Perspektive wurde in bisherigen TOD-Studien bislang nicht integriert. Die Dimension Development Potential ergänzt das Modell um die Erfassung von geeigneten Flächenpotenzialen zur (Weiter)entwicklung von TOD-Standorten. Wie in Tabelle 1 dargestellt, wurden Flächenpotenziale im Rahmen von TOD-Studien bisher nur von Seidemann (2016) erfasst.

Tabelle 2 gibt einen Überblick darüber, mit welchen Indikatoren die Operationalisierung der TOD-Dimensionen erfolgte, welche Analyseebene ausgewählt und mit welcher Datengrundlage gearbeitet wurde. Wie ebenfalls aus Tabelle $2 \mathrm{zu}$ entnehmen ist, wurden einzelne TOD-Dimensionen dabei über mehrere Indikatoren erfasst. Beispielsweise erfolgte die Operationalisierung der Dimension Destination Accessibility insgesamt auf der Basis von drei Indikatoren: 1) Taktfrequenz im Regional- und Fernverkehr, 2) Umsteigepotenzial im Einzugsbereich und 3) Umfang des regionalen Bevölkerungspotenzials. Andere TOD-Dimensionen wie Density lassen sich wiederum über einen einzelnen Indikator wie die Bevölkerungsdichte erfassen.

In den folgenden Abschnitten wird das generelle Vorgehen zur Operationalisierung der TOD-Dimensionen beschrieben.

\subsubsection{Destination Accessibility (D1)}

Die Taktfrequenz ist ein entscheidender Faktor, wenn es um eine möglichst unterbrechungsfreie Fahrt mit öffentlichen Verkehrsmitteln und die generelle Attraktivität von Haltepunkten geht. Bereits in Studien von Lyu, Bertolini und 
Tabelle 1 Einbezogene D's der TOD-Basisstudien

\begin{tabular}{|c|c|c|c|c|c|c|c|c|}
\hline \multirow{2}{*}{$\begin{array}{l}\text { Autorinnen/ } \\
\text { Autoren/Jahr }\end{array}$} & \multirow{2}{*}{$\begin{array}{l}\text { Stadt/ } \\
\text { Region }\end{array}$} & \multicolumn{7}{|l|}{ 6-D-Modell } \\
\hline & & $\begin{array}{l}\text { 1) Destina- } \\
\text { tion acces- } \\
\text { sibility }\end{array}$ & 2) Density & $\begin{array}{l}\text { 3) Diversi- } \\
\text { ty }\end{array}$ & $\begin{array}{l}\text { 4) Distance } \\
\text { to Transit }\end{array}$ & $\begin{array}{l}\text { 5) Demand } \\
\text { Manage- } \\
\text { ment }\end{array}$ & 6) Design & $\begin{array}{l}\text { 7) Deve- } \\
\text { lopment } \\
\text { Potential }\end{array}$ \\
\hline $\begin{array}{l}\text { Bahn.Ville 2-Kon- } \\
\text { sortium (2010) }\end{array}$ & $\begin{array}{l}\text { Rhein- } \\
\text { Main }\end{array}$ & $\bullet$ & $\bullet$ & & $\bullet$ & & & \\
\hline $\begin{array}{l}\text { Boarnet/Compin } \\
\text { (1999) }\end{array}$ & San Diego & & & $\bullet$ & & & & \\
\hline $\begin{array}{l}\text { Büttner/ } \\
\text { Kinigadner/Ji } \\
\text { et al. (2018) }\end{array}$ & München & $\bullet$ & $\bullet$ & $\bullet$ & $\bullet$ & & & \\
\hline $\begin{array}{l}\text { Cervero/ } \\
\text { Kockelman (1997) }\end{array}$ & $\begin{array}{l}\text { San Fran- } \\
\text { cisco }\end{array}$ & & $\bullet$ & $\bullet$ & & & $\bullet$ & \\
\hline Chen/Lin (2015) & Shanghai & $\bullet$ & $\bullet$ & $\bullet$ & & & & \\
\hline Curtis (2008) & Perth & & & $\bullet$ & & & & \\
\hline $\begin{array}{l}\text { García-Paloma- } \\
\text { res/Gutiérrez/ } \\
\text { Cardozo (2013) }\end{array}$ & Madrid & $\bullet$ & $\bullet$ & & & & & \\
\hline $\begin{array}{l}\text { Gilat/Sussman } \\
\text { (2003) }\end{array}$ & $\begin{array}{l}\text { Mexico } \\
\text { City }\end{array}$ & & $\bullet$ & & & & & \\
\hline $\begin{array}{l}\text { Greene/Mora/ } \\
\text { Figueroa et al. } \\
\text { (2017) }\end{array}$ & $\begin{array}{l}\text { Santiago } \\
\text { de Chile }\end{array}$ & & $\bullet$ & & & & & \\
\hline $\begin{array}{l}\text { Hickman/Hall } \\
(2008)\end{array}$ & London & & $\bullet$ & & & & & \\
\hline $\begin{array}{l}\text { Higgins/ } \\
\text { Kanaroglou (2016) }\end{array}$ & Toronto & & $\bullet$ & $\bullet$ & $\bullet$ & & & \\
\hline $\begin{array}{l}\text { Liang/Du/Wang } \\
\text { et al. (2020) }\end{array}$ & Jiaomei & & & $\bullet$ & & & & \\
\hline $\begin{array}{l}\text { Loo/Cheng/ } \\
\text { Nichols (2017) }\end{array}$ & Hongkong & & $\bullet$ & $\bullet$ & & & & \\
\hline $\begin{array}{l}\text { Lyu/Bertolini/ } \\
\text { Pfeffer (2016) }\end{array}$ & Peking & $\bullet$ & $\bullet$ & $\bullet$ & $\bullet$ & $\bullet$ & & \\
\hline $\begin{array}{l}\text { Monajem/ } \\
\text { Nosratian (2015) }\end{array}$ & Teheran & $\bullet$ & $\bullet$ & $\bullet$ & $\bullet$ & & & \\
\hline $\begin{array}{l}\mathrm{Niu} / \mathrm{Hu} / \mathrm{Shen} \\
\text { et al. (2019) }\end{array}$ & Singapur & & & $\bullet$ & & & & \\
\hline $\begin{array}{l}\text { Pitot/Yigitcanlar/ } \\
\text { Sipe et al. (2006) }\end{array}$ & $\begin{array}{l}\text { Gold } \\
\text { Coast } \\
\text { (Australi- } \\
\text { en) }\end{array}$ & $\bullet$ & & $\bullet$ & $\bullet$ & & & \\
\hline $\begin{array}{l}\text { Reusser/ } \\
\text { Loukopoulos/ } \\
\text { Stauffacher et al. } \\
\text { (2008) }\end{array}$ & Schweiz & $\bullet$ & $\bullet$ & $\bullet$ & $\bullet$ & & & \\
\hline Seidemann (2016) & $\begin{array}{l}\text { Baden- } \\
\text { Württem- } \\
\text { berg }\end{array}$ & $\bullet$ & & $\bullet$ & & & & $\bullet$ \\
\hline $\begin{array}{l}\text { Singh/Fard/ } \\
\text { Zuidgeest et al. } \\
\text { (2014) }\end{array}$ & $\begin{array}{l}\text { Arnheim, } \\
\text { Nijmegen }\end{array}$ & & & $\bullet$ & & & & \\
\hline $\begin{array}{l}\text { Strong/Ozbek/ } \\
\text { Sharma et al. } \\
\text { (2017) }\end{array}$ & Denver & & $\bullet$ & & $\bullet$ & $\bullet$ & $\bullet$ & \\
\hline
\end{tabular}


Tabelle 2 Operationalisierung der TOD-Dimensionen

\begin{tabular}{|c|c|c|c|c|c|}
\hline D's & Erläuterung & $\begin{array}{l}\text { Theoretischer } \\
\text { Wertebereich }\end{array}$ & (Teil-)Indikatoren & Analyseebene & Datengrundlage \\
\hline \multirow[t]{3}{*}{$\begin{array}{l}\text { Destination } \\
\text { Accessibility } \\
\text { (D1) }\end{array}$} & \multirow[t]{3}{*}{$\begin{array}{l}\text { Angebotsqualität des } \\
\text { Haltepunkts im } \\
\text { Gesamtnetz }\end{array}$} & \multirow[t]{3}{*}{$0-100$} & $\begin{array}{l}\text { Taktfrequenz im Regio- } \\
\text { nal- und Fernverkehr }\end{array}$ & $\begin{array}{l}\text { Haltepunkt } \\
\text { (node) }\end{array}$ & $\begin{array}{l}\text { GTFS, VRR, VRS, } \\
\text { AVV, NWL, DB } \\
\text { RE/FV (2020) }\end{array}$ \\
\hline & & & $\begin{array}{l}\text { Umsteigepotenzial auf } \\
\text { den lokalen Verkehr }\end{array}$ & $\begin{array}{l}\text { Einzugsbereich } \\
\text { (place) }\end{array}$ & $\begin{array}{l}\text { Zensusatlas } \\
\text { (2011) }\end{array}$ \\
\hline & & & $\begin{array}{l}\text { Regionales Bevölke- } \\
\text { rungspotenzial (normali- } \\
\text { siert) }\end{array}$ & $\begin{array}{l}\text { Haltepunkt } \\
\text { (node) }\end{array}$ & $\begin{array}{l}\text { Zensusatlas } \\
\text { (2011) }\end{array}$ \\
\hline Density (D2) & Dichte & $0-\infty$ & $\begin{array}{l}\text { Bevölkerungsdichte (in } \\
\text { Einwohner/ha) }\end{array}$ & $\begin{array}{l}\text { Einzugsbereich } \\
\text { (place) }\end{array}$ & $\begin{array}{l}\text { Zensusatlas } \\
\text { (2011) }\end{array}$ \\
\hline \multirow[t]{2}{*}{ Diversity (D3) } & \multirow{2}{*}{$\begin{array}{l}\text { Nutzungsmischung und } \\
\text { nahräumliche } \\
\text { Versorgung }\end{array}$} & \multirow[t]{2}{*}{$0-100$} & $\begin{array}{l}\text { Shannon-Index (normali- } \\
\text { siert) }\end{array}$ & $\begin{array}{l}\text { Einzugsbereich } \\
\text { (place) }\end{array}$ & ATKIS (2018) \\
\hline & & & Walkscore (normalisiert) & $\begin{array}{l}\text { Einzugsbereich } \\
\text { (place) }\end{array}$ & OSM (2020) \\
\hline $\begin{array}{l}\text { Distance to } \\
\text { Transit (D4) }\end{array}$ & $\begin{array}{l}\text { Verkehrliche Erreichbar- } \\
\text { keit des Haltepunkts }\end{array}$ & $0-\infty$ & $\begin{array}{l}\text { Länge des Fußwegenet- } \\
\text { zes (in m) }\end{array}$ & $\begin{array}{l}\text { Einzugsbereich } \\
\text { (place) }\end{array}$ & OSM (2020) \\
\hline $\begin{array}{l}\text { Demand } \mathrm{Ma-} \\
\text { nagement (D5) }\end{array}$ & Nachfragesteuerung & $0-\infty$ & $\begin{array}{l}\text { Parkplatzfläche (in } \mathrm{m}^{2} \text { ) } \\
\text { Vorhandensein von } \\
\text { Park+Ride }\end{array}$ & $\begin{array}{l}\text { Einzugsbereich } \\
\text { (place) }\end{array}$ & OSM (2020) \\
\hline $\begin{array}{l}\text { Development } \\
\text { Costs (D6) }\end{array}$ & Entwicklungskosten & $0-\infty$ & $\begin{array}{l}\text { Bodenrichtwerte (in } \\
\text { Euro } / \mathrm{m}^{2} \text { ) }\end{array}$ & $\begin{array}{l}\text { Einzugsbereich } \\
\text { (place) }\end{array}$ & $\begin{array}{l}\text { BORIS.NRW } \\
(2018)\end{array}$ \\
\hline $\begin{array}{l}\text { Development } \\
\text { Potential (D7) }\end{array}$ & Entwicklungspotenzial & $0-\infty$ & $\begin{array}{l}\text { Entwickelbares Flächen- } \\
\text { potenzial (in ha) }\end{array}$ & $\begin{array}{l}\text { Einzugsbereich } \\
\text { (place) }\end{array}$ & ATKIS (2018) \\
\hline
\end{tabular}

GTFS: General Transit Feed Specification, VRR: Verkehrsverbund Rhein-Ruhr, VRS: Verkehrsverbund Rhein-Sieg, AVV: Aachener Verkehrsverbund, NWL: Zweckverband Nahverkehr Westfalen-Lippe, DB RE/FV: DB Open-Data-Portal, ATKIS: Amtliches TopographischKartographisches Informationssystem, OSM: OpenStreetMap, BORIS.NRW: Bodenrichtwerte Nordrhein-Westfalen

Pfeffer (2016) oder Reusser, Loukopoulos, Stauffacher et al. (2008) wurde die Taktfrequenz als ein essenzieller Bestandteil für eine erfolgreiche TOD-Implementierung bewertet. Die Taktfrequenz wird in der vorliegenden Studie daher ebenfalls integriert und differenziert nach Fernverkehr (IC und ICE) und Nahverkehr (RE, RB, S-Bahn) operationalisiert. Die Taktfrequenzen werden über die jeweiligen Fahrplandaten der Verkehrsverbünde (VRR, VRS, AVV, NWL) berechnet.

Das Umsteigepotenzial im Einzugsbereich dient als Proxy, um die Vernetzung des Haltepunktes mit Verkehrsmitteln des lokalen Verkehrs abzubilden. Hierzu werden alle U-Bahn- und Tram- sowie Bushaltepunkte in den Einzugsbereichen entsprechend ihrer spezifischen Taktfrequenz betrachtet. Die Standorte und Fahrpläne der Haltepunkte sind ebenfalls den öffentlichen Datenbanken der Verkehrsverbünde entnommen. In Anlehnung an Anderhub, Dorbritz und Weidmann (2008), die aus der Kombination von Fassungsvermögen der Fahrzeuge und Taktdichte eine etwa doppelt so hohe Leistungsfähigkeit von U- und Tram-Bahnen gegenüber Bussen für die Fallstudienstädte Zürich und Bern ermitteln konnten, werden diese Haltepunkte in der vorliegenden Analyse vereinfachend doppelt gewichtet. Eine ähnliche Annahme wird auch im ,Monitoring Daseins- vorsorge 2017“ des Regionalverbands Ruhr zur Berechnung des dort verwendeten Haltestellen-Index getroffen (Alfken/ Iwer 2017: $52 \mathrm{ff}$.). Da weit entfernte Haltepunkte weniger attraktiv für einen Umstieg sind, wird zudem eine Gehzeitbasierte Gewichtung der Haltepunkte verwendet. Der maximale Punktwert für das Umsteigepotenzial pro Einzugsbereich ergibt sich demnach aus Anzahl, Art und Entfernung der Haltepunkte im Einzugsbereich. Da davon ausgegangen werden kann, dass der Grenznutzen weiterer Haltepunkte ab einem bestimmten Niveau gegen Null tendiert, beinhaltet die Berechnung einen theoretischen maximalen Sättigungsgrad pro Einzugsbereich.

Das regionale Bevölkerungspotenzial ist ein klassisches Maß der Bevölkerungsgeographie, um die Möglichkeit von Interaktionen im Raum darzustellen (Maretzke 2014: 16f.). Hierzu wird ermittelt, wie viele Menschen innerhalb einer bestimmten Zeit bzw. Entfernung von einem Ort erreicht werden können. Umso mehr Menschen erreicht werden können, desto höher fällt das Potenzial aus. Im Rahmen der Analyse wird das Maß dazu verwendet, um die Bedeutung eines Haltepunktes im regionalen Kontext (z. B. Erreichbarkeit von zentralen Orten) abzubilden. Das Potenzial wird als Anzahl der Einwohner/-innen berechnet, die in 45 Minuten Fahrzeit mit der Bahn inklusive zwei Kilome- 
ter Fußweg (sofern 45 Minuten nicht überschritten werden) erreicht werden können. Grundlage für die Ermittlung des Potenzials ist das hochauflösende Zensus-Grid (100x100m) von 2011 und die Software OpenTripPlanner, mit der die Fahrzeitisochrone berechnet wurden. Aus Autorensicht wären darüber hinaus regionale Beschäftigtenpotenziale oder regional bedeutsame Bildungs- und Freizeiteinrichtungen und Einrichtungen der Daseinsvorsorge wichtige Aspekte, um die Erreichbarkeitsbewertung der Haltepunkte weiter zu verfeinern. Flächendeckende, kleinräumige Daten zu Beschäftigten oder spezifischen Einrichtungen liegen aber nicht vor oder weisen teils erhebliche Datenlücken und -fehler auf, so dass in der Analyse lediglich auf die Bevölkerungsdaten zurückgegriffen wurde.

\subsubsection{Density (D2)}

Höhere Bevölkerungsdichten im Umfeld von Haltepunkten sind aus zwei Gründen relevant: Erstens nutzen Menschen, die in der Nähe von Haltepunkten wohnen, häufiger den öffentlichen Nahverkehr. Hierdurch verbessert sich zum einen dessen Tragfähigkeit (Ibraeva/Correia/Silva et al. 2020: $114 \mathrm{ff}$.) und zum anderen erfolgt die Mobilität der Menschen nachhaltiger. Zweitens bieten bereits bestehende Bevölkerungsdichten einen wichtigen Anhaltspunkt darüber, wie dicht zukünftige Wohnbauvorhaben realisiert werden können. Höhere Dichten fördern einen effizienteren Ressourceneinsatz und können genutzt werden, um hohe Bodenpreise, insbesondere in den innerstädtischen Lagen, zu kompensieren (Schmitt/Seidl 2006: 97 ff.). Zur Ermittlung spezifischer Dichtewerte werden die hochauflösenden Bevölkerungsdaten aus dem Zensus-Grid (100x100m) verwendet. Diese Zahlen werden dabei auf die in den Einzugsbereichen befindlichen Klassen Mischnutzung und Wohnnutzung aus dem Amtlichen Topographisch-Kartographischen Informationssystem (ATKIS) umverteilt.

\subsubsection{Diversity (D3)}

Die Durchmischung der Einzugsbereiche wird als bedeutendes Kriterium für eine erfolgreiche TOD-Implementierung angesehen. Eine ausreichend hohe Vielfalt an Angeboten ermöglicht es der Bevölkerung, unterschiedliche Bedürfnisse im direkten Umfeld fußläufig zu befriedigen, was die Attraktivität der entsprechenden Räume steigert. Im Rahmen der vorliegenden Studie wird die Nutzungsmischung der Einzugsbereiche zum einen über den Shannon-Index (Shannon/Weaver 1964) und zum anderen über den Walkscore $^{4}$ abgeleitet. Der Shannon-Index ist ein Diversitätsmaß, mit dem die Durchmischung der Nutzung durch einen ein-

\footnotetext{
4 http://pubs.cedeus.cl/omeka/files/original/b6fa690993d5900 7784a7a26804d42be.pdf (08.12.2020).
}

zelnen Indexwert ausgedrückt werden kann. Der Index wird auf der Basis der ATKIS-Flächennutzungsklassen ermittelt und gibt an, wie divers die Struktur der Flächennutzung ist. Der Walkscore gibt an, wie gut die fußläufige Erreichbarkeit zu wichtigen Einrichtungen der Daseinsvorsorge an einem Standort ist. Dazu werden die fußläufigen Distanzen vom Haltepunkt zu Infrastrukturen aus dem Bereich Bildung, Gesundheit, Einrichtungen des täglichen Bedarfs, Einrichtungen des sonstigen Bedarfs sowie Freizeit und Kultur berechnet. Je geringer die Distanz, desto höher liegt die Bewertung der Infrastruktur. Bei Infrastrukturen, die mehr als $2.500 \mathrm{~m}$ entfernt sind, wird davon ausgegangen, dass diese für das fußläufige Aufsuchen nicht mehr attraktiv sind. Die einzelnen Infrastrukturkategorien werden je nach Bedeutung für das alltägliche Leben höher oder niedriger gewichtet. Daten zum Straßennetz und den einzelnen Einrichtungen wurden aus OpenStreetMap (OSM) abgerufen.

\subsubsection{Distance to Transit (D4)}

Die Zugänglichkeit der Haltepunkte für Fußgänger/-innen wird in der TOD-Literatur, aber auch in der Literatur zum Urban Design als ein wichtiges Kriterium für einen attraktiven Haltepunkt bewertet (Jamme/Rodriguez/Bahl et al. 2019). Cervero und Kockelman (1997) konnten anhand von Indikatoren zum Straßennetz oder zur Verkehrsinfrastruktur für Fußgänger/-innen und Radfahrer/-innen einen statistisch signifikanten Einfluss auf das Reisen ohne den Pkw nachweisen. Im Rahmen der vorliegenden Studie erfolgt die Operationalisierung dieser Dimension vereinfachend über die Länge des Fußwegenetzes im Einzugsbereich der Haltepunkte. Der Argumentation von Cervero und Kockelman (1997) folgend, gehen die Autoren davon aus, dass ein dichteres Fußwegenetz das Zufußgehen und Fahrradfahren insgesamt attraktiver macht. Obwohl damit keine Faktoren wie Zustand der Gehwege, Sauberkeit oder Lärmbelastung erfasst werden, bietet der Indikator dennoch eine erste Annäherung, um die Erreichbarkeit und Fußgängerfreundlichkeit eines Haltepunktes indirekt zu operationalisieren.

\subsubsection{Demand Management (D5)}

Unter der Dimension Demand Management wird die Steuerung der Nachfrage durch bauliche und nichtbauliche Maßnahmen zusammengefasst. In der Literatur wird hierbei unter anderem die Reduzierung von Parkplätzen im Wohnumfeld (Cervero/Arrington 2008), aber auch das Parken im direkten Umfeld der TOD-Haltepunkte (z. B. Park+Ride) diskutiert, um den Umstieg auf den ÖPNV zu vereinfachen (Tumlin/Millard-Ball 2006; Ogra/Ndebele 2014). Die Operationalisierung dieser Dimension erfolgt über die Erfassung aller Parkplatzflächen (in ha) in einem Radius von 200 m um den Haltepunkt. Zusätzlich hierzu werden Park+Ride-Stationen erhoben. 
Tabelle 3 Deskriptive Statistiken der TOD-Dimensionen

\begin{tabular}{lllllllll}
\hline & $\mathbf{n}$ & $\begin{array}{l}\text { Mittel- } \\
\text { wert }\end{array}$ & $\begin{array}{l}\text { Standard- } \\
\text { abweichung }\end{array}$ & Median & $\begin{array}{l}\text { Minimal- } \\
\text { wert }\end{array}$ & $\begin{array}{l}\text { Maximal- } \\
\text { wert }\end{array}$ & $\begin{array}{l}\text { Spannweite } \\
\text { Standard- } \\
\text { fehler }\end{array}$ \\
\hline $\begin{array}{l}\text { Destination Accessibility } \\
\text { (D1) }\end{array}$ & 747 & 41,3 & 22,8 & 37,5 & 0,4 & 100,0 & 99,6 & 0,8 \\
$\begin{array}{l}\text { Density (D2) } \\
\text { Diversity (D3) }\end{array}$ & 747 & 35,9 & 37,7 & 25,8 & 0,0 & 786,3 & 404,2 & 1,4 \\
$\begin{array}{l}\text { Distance to Transit (D4) } \\
\text { Demand Management }\end{array}$ & 747 & 47,2 & 15,8 & 49,9 & 5,2 & 80,2 & 75,0 & 0,6 \\
$\begin{array}{l}\text { (D5) } \\
\text { Development Costs (D6) }\end{array}$ & 747 & 0,7 & 0,8 & 0,4 & 0,0 & 5,8 & $38.088,7$ & 190,9 \\
$\begin{array}{l}\text { Development Potential } \\
\text { (D7) }\end{array}$ & 747 & 270,3 & 37,9 & $5.212,8$ & $5.309,3$ & 73,0 & $38.161,7$ & 0,0 \\
\end{tabular}

Die Geometrie der Parkplatzflächen ist OSM entnommen, die Standorte zu Park+Ride-Stationen stammen von Google Maps. Durch die räumliche Überlagerung der Informationen werden die Parkplatzflächen identifiziert, die als Park+Ride genutzt werden. Um die Bedeutung von Park+Ride für TOD-Haltepunkte hervorzuheben, werden diese Flächen mit 2 multipliziert.

\subsubsection{Development Costs (D6)}

Hohe Bodenpreise führen zu einer intensiveren Flächennutzung und fördern verdichtetes Bauen, werden aber auch durch Investoren über die Miet- und Kaufpreise refinanziert (Schmitt/Seidl 2006: $97 \mathrm{ff}$.; Spars 2017: $79 \mathrm{ff.}$.). Bodenpreise in den Einzugsbereichen von Haltepunkten können daher als Proxy genutzt werden, um das Spannungsfeld zwischen der Schaffung von lockerer und verdichteter Bebauung sowie bezahlbarem und höherpreisigem Wohnen abzubilden. Der in der Analyse berücksichtigte zonale Bodenrichtwert (zBRW) ist BORIS.NRW ${ }^{5}$ entnommen und stellt einen durchschnittlichen Wert für den Boden dar, der Ausdruck der lagespezifischen Wertschätzung einer baulichen Nutzungsmöglichkeit ist. Beeinflusst wird der Marktpreis durch Standortqualitäten und Standortbeeinträchtigungen sowie durch das Verhältnis von Angebot und Nachfrage. Er wird in Euro/ $\mathrm{m}^{2}$-Grundstücksfläche angegeben und in einer Richtwertzone ausgewiesen.

\subsubsection{Development Potential (D7)}

Zur Identifikation des Umfangs potenziell entwicklungsfähiger Flächen werden die flächenhaften Darstellungen aus ATKIS verwendet. Als Potenzial gelten alle Flächen, die nicht baulich geprägt, als Gewässer klassifiziert sind oder nicht forstlich genutzt werden. Die Verwendung der flächenhaften ATKIS-Daten hat zur Folge, dass Nachverdich-

\footnotetext{
${ }^{5}$ https://www.boris.nrw.de (08.12.2020).
}

tungspotenziale (z. B. Baulücken) mit der aktuellen Methodik nicht berücksichtigt werden können. Aus Autorensicht wird mit dem engen räumlichen Fokus (fußläufige Erreichbarkeit) um die Haltepunkte aber gewährleistet, dass vor allem solche Flächenpotenziale Berücksichtigung finden, die als integriert gelten können. Dennoch bietet die Erfassung von Nachverdichtungspotenzialen zukünftig eine wichtige methodische Weiterentwicklungsmöglichkeit. Nach aktuellem Stand der Technik und in Anlehnung an Paulsen (2014) werden weiterhin Flächen mit einer Hangneigung von mehr als $15^{\circ}$ als nicht (wirtschaftlich) bebaubar eingestuft und nicht berücksichtigt. Da es bei der Analyse insbesondere um die Identifizierung von regional bedeutsamen Potenzialen geht, werden des Weiteren Flächen mit einer Größe von unter einem Hektar ausgeschlossen.

Mithilfe der erhobenen Indikatoren bzw. TOD-Dimensionen wird eine hierarchische Clusteranalyse durchgeführt, mit der die Haltepunkte entsprechend ihrer Ausprägung pro TOD-Dimension für eine an den schienengebundenen Regionalverkehr ausgerichtete Siedlungsentwicklung klassifiziert werden. Die Clusteranalyse gruppiert den Stichprobensatz der 747 Haltepunkte so, dass die Varianz der Indikatoren innerhalb der Cluster minimal und zwischen den Clustern maximal ist. Hierzu werden die Indikatoren zunächst z-standardisiert, um die Gewichtung aller Indikatoren in der Analyse gleich zu halten. Die Cluster selbst werden mithilfe der euklidischen Distanzmessung und der Ward-Fusionierungsmethode gebildet. Die Berechnung wird mittels der Software R Studio durchgeführt.

\section{Ergebnisse}

Tabelle 3 gibt einen Überblick über die deskriptiven Statistiken der in dieser Studie abgebildeten TOD-Dimensionen. Die ermittelten Wertespannen zeigen, dass es in allen Dimensionen eine nicht unerhebliche Varianz zwischen den analysierten Haltepunkten gibt, was für eine hohe Hetero- 


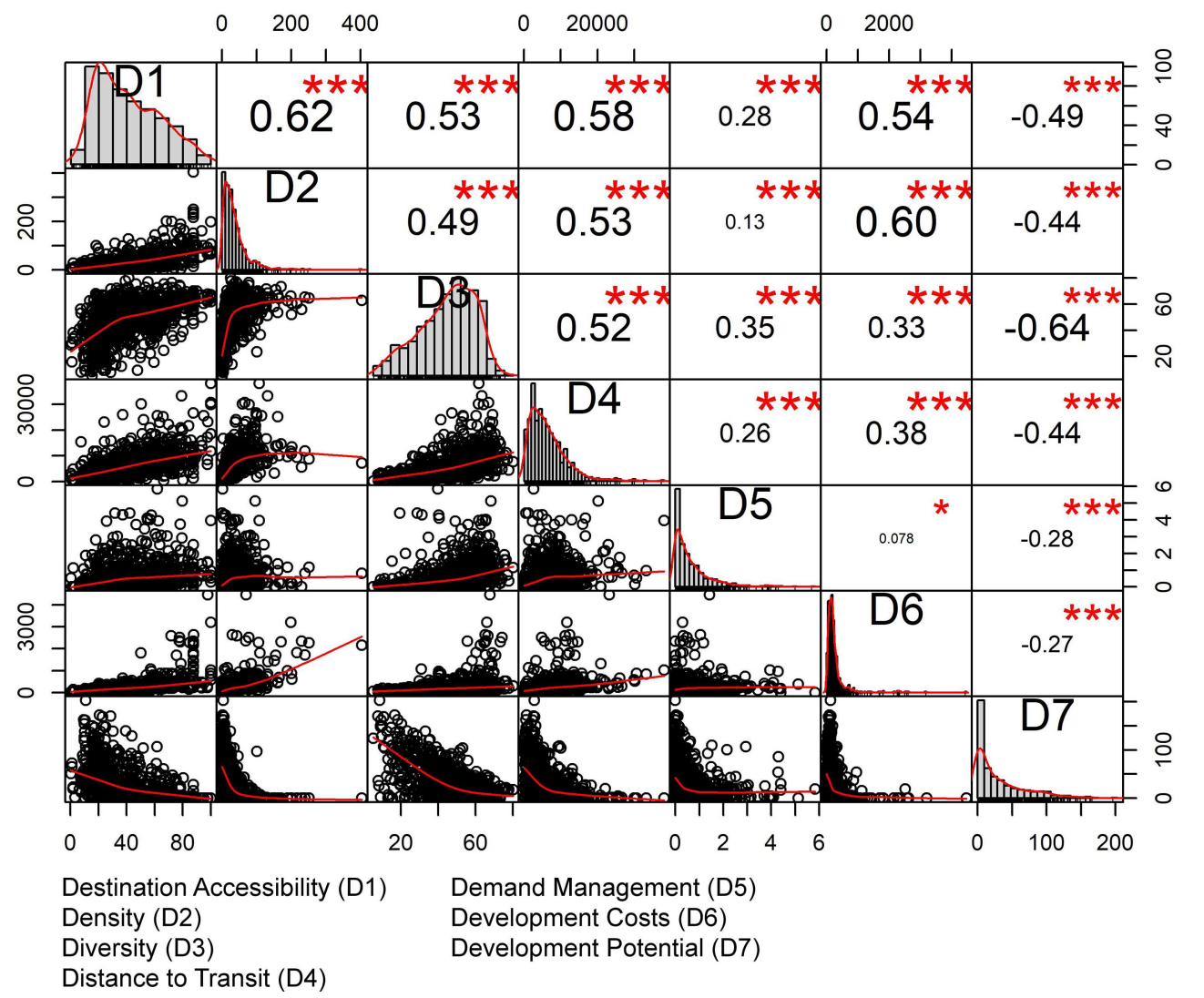

Abbildung 1 Korrelationsmatrix zu den Indikatoren der TOD-Dimensionen

Anmerkung: ${ }^{* * *} p=0.00,{ }^{*} p=0.05$

genität zwischen den Haltepunkten spricht. Insbesondere bei den Dimensionen Density, Distance to Transit und Development Costs wird dies deutlich.

Abbildung 1 zeigt die Verteilung der einzelnen Variablen in der Diagonalen. Unterhalb der Diagonalen werden die bivariaten Streudiagramme und oberhalb der Diagonalen die Werte der Korrelationen sowie die Signifikanzniveaus zwischen den TOD-Dimensionen dargestellt. Die Stärke der Korrelationen variiert hier zwischen -0,64 und +0,62. Es besteht ein mittlerer bis starker Zusammenhang insbesondere zwischen den TOD-Dimensionen Destination Accessibility, Density, Diversity, Distance to Transit und Development Costs. Haltepunkte, die eine hohe Angebotsqualität aufweisen, sind somit oft auch durch höhere Dichten, eine gute nahräumliche Versorgung und Nutzungsdurchmischung sowie ein gut ausgebautes Fußwegenetz geprägt und umgekehrt. Die TOD-Dimension Demand Management korreliert dahingegen nur auf einem niedrigen Niveau mit den anderen TOD-Dimensionen. Zwischen der TOD-Dimension Development Potential und allen anderen Dimensionen besteht eine negative Korrelation. Der negative Zusammenhang zeigt, dass beispielsweise mit einer höheren Angebotsqualität oder höheren Dichtewerten der Umfang der entwi- ckelbaren Flächen abnimmt. Abbildung 1 zeigt zudem, dass die Korrelationen zwischen den TOD-Dimensionen statistisch hochsignifikant sind. Das Signifikanzniveau liegt in 20 von 21 Fällen bei $\mathrm{p}=0$. Lediglich zwischen Demand $M a$ nagement und Development Costs wird nur ein Signifikanzniveau von $\mathrm{p}=0,05$ erreicht.

Abbildung 2 zeigt die Mittelwerte pro TOD-Dimension und Cluster sowie den Mittelwert pro TOD-Dimension über die gesamte Stichprobe. Die Abbildung ermöglicht eine Abschätzung darüber, ob das Cluster im Vergleich zur gesamten Stichprobe über- oder unterdurchschnittlich abschneidet. In der vorliegenden Studie ergaben verschiedene Testverfahren (Ellbogenkriterium und Silhouettenkoeffizient) zur Bestimmung der optimalen Cluster-Anzahl Lösungen zwischen 3 und 5 Cluster (vgl. Kodinariya/Makwana 2013). Um eine klarere Abgrenzung zwischen den Clustern zu erreichen, wurde die Fünf-Cluster-Lösung gewählt.

Wie in Abbildung 2 zu sehen ist, besteht bei der 5-Cluster-Lösung das Cluster $1(n=17)$ aus Haltepunkten mit überwiegend überdurchschnittlichen Werten pro TOD-Dimension. Augenfällig für dieses Cluster ist der im Vergleich sehr hohe Durchschnittswert bei den Development Costs sowie der sehr niedrige Durchschnittswert beim Development 


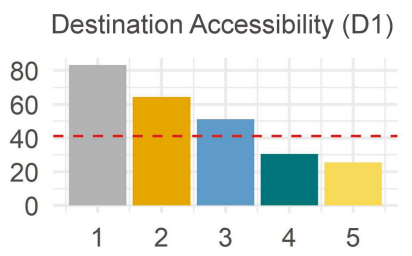

Distance to Transit (D4)

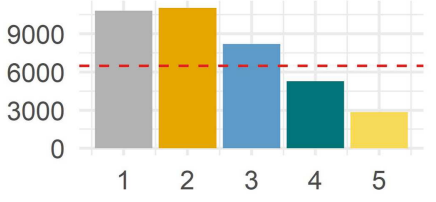

Development Potential (D7)

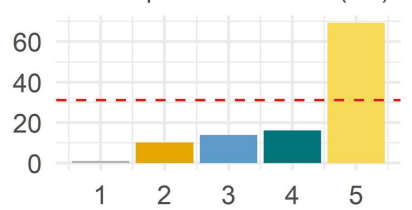

Abbildung 2 Mittelwerte pro TOD-Dimensionen und Cluster

Potential. Das Cluster $2(\mathrm{n}=233)$ umfasst Haltepunkte mit ebenfalls mehrheitlich überdurchschnittlichen Werten pro TOD-Dimension. Das Cluster unterscheidet sich von Cluster 1 vor allem durch den deutlich niedrigeren Wert in der TOD-Dimension Development Costs, der im Vergleich zur gesamten Stichprobe zwar noch überdurchschnittlich ausfällt, aber nur ein Fünftel des Wertes von Cluster 1 erreicht. Das Cluster $3(n=26)$ kommt ebenfalls auf überdurchschnittliche Werte, diese fallen aber weniger deutlich aus als bei Cluster 1 und 2. Abbildung 2 zeigt, dass der Treiber für dieses Cluster die TOD-Dimension Demand Management ist, die im Vergleich zu den anderen Clustern hier besonders hervorsticht. Die Wertespanne dieser TOD-Dimension fällt generell gering aus. Cluster $4(n=229)$ ist durch unterdurchschnittliche Werte pro TOD-Dimension geprägt. Ausschließlich die TOD-Dimensionen Diversity und Demand Management fallen leicht überdurchschnittlich aus. Cluster $5(n=242)$ umfasst vor allem Haltepunkte mit deutlich unterdurchschnittlichen Werten pro TOD-Dimension. Wird die Dimension Development Potential ausgeklammert, werden hier in allen TOD-Dimensionen die niedrigsten Werte erreicht. Mit Ausnahme von der TOD-Dimension Demand Management zeigt sich, dass die Durchschnittswerte der TOD-Dimensionen Destination Accessibility, Density, Diversity, Distance to Transit und Development Costs gegenläufig zu den Durchschnittswerten der TOD-Dimension Development Potential verlaufen. Dies lässt den Schluss zu, dass der Umfang potenziell entwickelbarer Flächen mit Zunahme der anderen TOD-Dimensionen insgesamt abnimmt.

Überträgt man die gebildeten Cluster in eine kartographische Darstellung, zeigt sich ein deutliches Stadt-LandGefälle ausgehend von Cluster 1. Die Haltepunkte aus Clus-

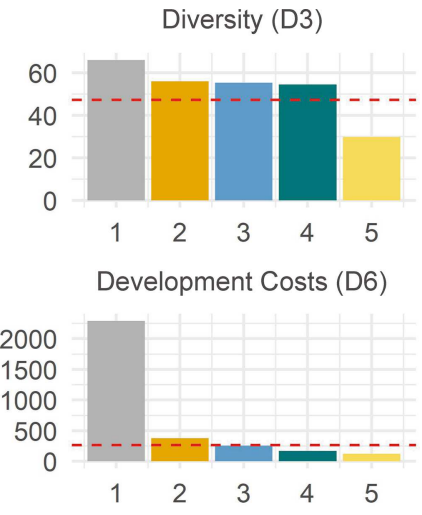

ter 1 finden sich ausschließlich in den innerstädtischen Lagen der sehr dynamisch wachsenden Kernstädte Münster, Bonn und Köln. Das Cluster 2 umfasst die übrigen weniger zentralen Haltepunkte dieser Städte sowie Haltepunkte in den anderen Kernstädten und deren Umland. Insbesondere im Rhein-Ruhr-Raum sowie in den solitären Städten Aachen, Münster, Bielefeld und Paderborn finden sich Haltepunkte dieses Clusters. Haltepunkte aus Cluster 3 liegen regelmäßig an größeren Einzelhandels-, Gewerbe- oder Industriestandorten sowie größeren Parkplätzen mit Stationen für Park+Ride. Insgesamt lassen sich diese Haltepunkte vorwiegend in städtischen Randlagen oder peripheren Lagen finden. Haltepunkte, die den Clustern 4 und 5 zugeordnet sind, sind vorrangig im peripheren Raum lokalisiert. Erfasst werden durch diese Cluster vor allem die (sehr) kleinen Haltepunkte zwischen den größeren Verkehrsknotenpunkten. Abbildung 3 zeigt anhand von fünf Haltepunkten exemplarisch die Bebauungsstruktur der jeweiligen Cluster.

\section{Schlussfolgerung und Diskussion}

Unter Berücksichtigung der hier operationalisierten TODDimensionen sind die Haltepunkte der Cluster 1 und 2 besonders geeignet für eine am Schienenverkehr ausgerichtete Siedlungsentwicklung. Entsprechende Haltepunkte bieten eine hohe Angebotsqualität, urbane Dichten, eine gute nahräumliche Versorgung und Nutzungsdurchmischung sowie ein gut ausgebautes Fußwegenetz. Es zeigt sich aber, dass es gerade diese Haltepunkte sind, die besonders hohe Bodenrichtwerte besitzen und ein besonders geringes Flächenpotenzial aufweisen. Viele der Haltepunkte sind - aus- 

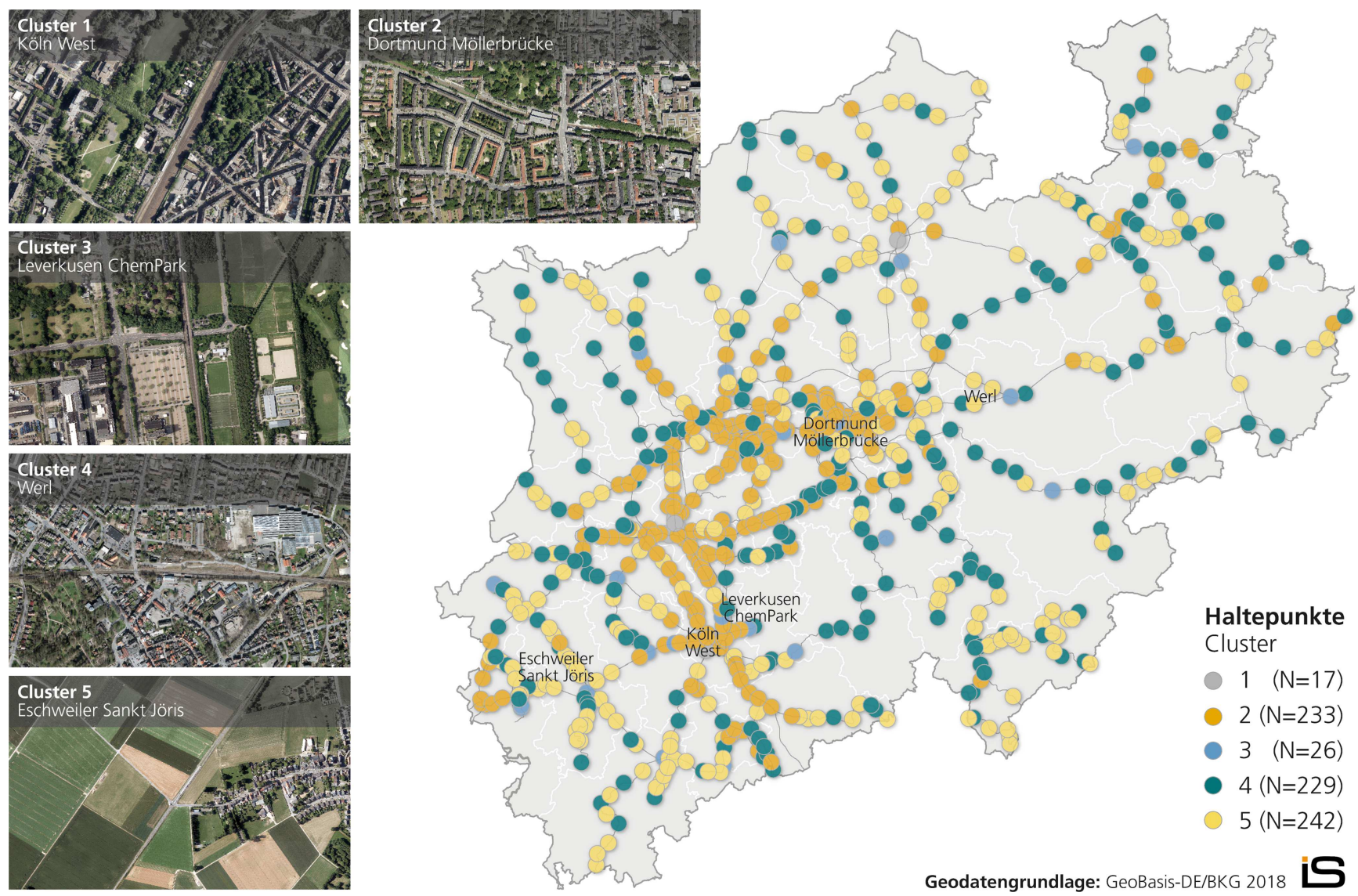

Geodatengrundlage: GeoBasis-DE/BKG 2018

Abbildung 3 Räumliche Verteilung der Cluster

gehend von der hier verwendeten Datengrundlage - bereits vollständig aufgesiedelt. Größere Siedlungsentwicklungen sind an diesen Haltepunkten daher nicht (mehr) möglich. Was an diesen Haltepunkten dahingegen denkbar ist, ist die Umsetzung von Maßnahmen der Nachverdichtung. Sowohl die Entwicklung von Brachflächen oder mindergenutzten Flächen als auch das Schließen von Baulücken oder das Aufstocken von Gebäuden wären Maßnahmen, die im Detail vor Ort geprüft werden könnten. Für größere Siedlungsentwicklungen kommen vor allem die Cluster 3, 4 und 5 in Frage. Im Vergleich zu den stark verdichteten innerstädtischen Haltepunkten finden sich hier viele und größere zusammenhängende entwickelbare Flächen. Zu beachten ist aber, dass diese Haltepunkte von einer - verglichen mit den anderen Clustern - eher unterdurchschnittlichen Angebotsqualität sowie einer unterdurchschnittlichen nahräumlichen Versorgung und Nutzungsdurchmischung geprägt sind. Die Schaffung von Wohnraum müsste daher mit flankierenden verkehrlichen und städtebaulichen Maßnahmen einhergehen, damit diese Standorte ihre Funktion im Sinne von Transit-Oriented Development wahrnehmen können. Mögliche Maßnahmen wären beispielsweise Taktverbesserungen des Regionalverkehrs oder die Ausweitung von Einrichtungen der Daseinsvorsorge im Einzugsbereich der Haltepunkte. Ein weiterer wichtiger Aspekt bei der Entwicklung entsprechender Standorte ist die wirtschaftliche Tragfähigkeit. Diese Frage stellt sich insbesondere bei Haltepunkten in sehr peripheren Räumen, wo gegebenenfalls hohe Kosten für die Schaffung und Bereitstellung von (neuen) sozialen und technischen Infrastrukturen anfallen können. Gerade vor dem Hintergrund geringerer Bevölkerungszahlen sollten Erschließungs- und Betriebskosten den möglichen steuerlichen Einnahmen des Bauvorhabens gegenübergestellt werden, um zu prüfen, ob eine wirtschaftliche Tragfähigkeit erreicht wird und langfristig gewährleistet werden kann. Dies ist vor allem auch deshalb von Bedeutung, da Flächenpotenziale verbreitet dort anzutreffen sind, wo die Nachfrage nach Bauland gering ausfällt. So lässt die Lage eines Haltepunktes weit ab von den größeren Verdichtungskernen häufig nur eine geringe lokale und regionale Nachfrage nach Wohnraum vermuten. Diese Angebot-NachfrageDisparitäten machen die Umwandlung von Potenzialflächen in Wohnbauflächen nicht immer und an jedem Ort sinnvoll.

Die Klassifizierung von TOD-Haltepunkten inklusive der Erfassung von Flächenpotenzialen kann zudem Eingang in 
die Revision von Regionalplänen nehmen. In Einzelfällen kann eine neue Abwägung sinnvoll sein, um mehr Bauland in infrastrukturell gut integrierten Lagen bereitzustellen. Für die städtebauliche Entwicklungsplanung bietet die Methode Möglichkeiten für strategische Priorisierungen und teilquantifizierte Szenarien. So können Modellrechnungen und Studien durchgeführt werden, an welchen Standorten welche Potenziale erschlossen werden könnten, wie attraktiv diese Potenziale sind und mit welchen Bodenpreisen im Rahmen kommunaler Liegenschaftspolitiken zu rechnen ist. Auch der strategische Erwerb von Liegenschaften (,,strategische Flächenreserve“) mit längerfristigen Entwicklungsoptionen der betreffenden Flächen ließe sich mit der Methode fachlich unterstützen.

Vor diesem Hintergrund würde sich ein systematisches Monitoring von TOD-Potenzialen und eine laufende Evaluierung der Mobilisierungsbemühungen entsprechender Flächen auf regionaler Ebene anbieten. Hierbei erscheint eine Vollerhebung der Potenziale alle drei bis fünf Jahre sinnvoll, um den Erfolg einer TOD-Politik transparent bewerten zu können, aber auch um aktuelle Planungsgrundlagen für die Praxis bereitzustellen. Insgesamt kann der Aufwand zur Implementierung eines solchen Ansatzes in die bestehende Raumbeobachtung als vergleichsweise gering eingeschätzt werden. Die Übertragbarkeit der Methodik auf andere Bundesländer schätzen die Autoren ebenfalls als gegeben ein. Bei der Methode kommen neben den amtlichen ATKIS-Daten, die den Ländern in der Regel frei zur Verfügung stehen, ausschließlich offene Daten (Open Data) zum Einsatz, was eine flexible Anwendung und Anpassung ermöglicht. Mit dem zunehmenden Bedeutungsgewinn offener und kleinräumiger Daten (z. B. Fahrplandaten) kann zudem davon ausgegangen werden, dass die Methode in den kommenden Jahren kontinuierlich weiterentwickelt werden kann, so auch zum Beispiel durch neue Datenprodukte aus der Fernerkundung, die es zukünftig ermöglichen, Innenentwicklungspotenziale besser zu identifizieren.

Abschließend sei angemerkt, dass die Ergebnisse der vorliegenden Studie gewissen Einschränkungen unterliegen. Diese sollen an dieser Stelle in aller Kürze dargestellt werden. So sind die ermittelten Flächenpotenziale in erster Linie als ein Annäherungswert zu verstehen. Neben topographischen oder naturräumlichen Einschränkungen, wie sie in dieser Studie berücksichtigt wurden, können viele weitere Faktoren die tatsächliche Mobilisierung von Flächen beeinflussen. Faktoren wie das Natur- und Umweltschutzrecht oder immissionsschutzrechtliche Vorgaben sowie planerische Überlegungen können dazu führen, dass in der Realität an Standorten deutlich geringere Flächenpotenziale bauleitplanerisch entwickelbar sind bzw. entwickelt werden. Wie bereits in Kapitel 3.2 angeführt, kommt hinzu, dass auf Basis der ATKIS-Daten ausschließlich unbebaute Flächen als
Potenzialflächen erfasst werden können. Nachverdichtungspotenziale wie Baulücken sind demzufolge in den Ergebnissen aktuell nicht enthalten. Die Ergebnisse werden zudem durch die Qualität und Aktualität der zugrunde liegenden Daten beeinflusst. Insbesondere bei der Ermittlung des regionalen Bevölkerungspotenzials kann lediglich auf die hochauflösenden Daten des Zensus-Atlas von 2011 zurückgegriffen werden. Dieser zeitliche Versatz kann zu Ungenauigkeiten des Potenzials führen. Aktuellere Daten stehen erst mit Abschluss des Zensus 2021 zur Verfügung. Während mit dem Zensus-Atlas bundesweit einheitliche hochauflösende Bevölkerungsdaten vorliegen, trifft dies auf die Nachbarstaaten nicht zu. Bei der Ermittlung des Bevölkerungspotenzials blieben die Nachbarländer und -staaten daher unberücksichtigt. Insbesondere in den Grenzregionen kann dies zu Verzerrungen und niedrigeren Werten führen.

\section{Fazit und weiterer Forschungsbedarf}

Die vorliegende Studie leistet einen Beitrag zur besseren Erfassung geeigneter TOD-Standorte sowie zur Förderung einer effizienteren, stadtregionalen Umsetzung von TODMaßnahmen in Nordrhein-Westfalen. Insgesamt zeigen die Ergebnisse, dass das TOD-Potenzial auch in einem hoch verdichteten, stark urbanisierten Raum wie Nordrhein-Westfalen noch nicht ausgeschöpft ist. Gleichwohl wird deutlich, dass im Umfeld der Haltepunkte der hochleistungsfähigen Bahnstrecken in Regionen mit hoher Wohnungsnachfrage eine ausgeprägte Flächenknappheit vorliegt, die mannigfaltige Nutzungskonflikte erwarten lässt.

In methodischer Hinsicht wird auch im internationalen Vergleich Neuland betreten, als innerhalb des Node-PlaceAnsatzes von den idealtypischen sechs TOD-Dimensionen fünf abgedeckt werden konnten. Weiterhin wurden zwei weitere - aus Sicht der Autoren - wichtige Dimensionen dem 6-D-Modell hinzugefügt. Sowohl die Erfassung von Flächenpotenzialen wie auch die Abschätzung der (Folge)kosten von Siedlungsentwicklungen in den Einzugsbereichen der Haltepunkte ermöglichen eine tiefergehende Bewertung und Abschätzung über die TOD-Eignung von spezifischen Standorten. Die Klassifizierung der Haltepunkte ergab für Nordrhein-Westfalen eine interpretierbare FünfCluster-Lösung, die den Diskussionsprozess im Hinblick auf das Finden von Vorschlägen für die weitere Entwicklung dieser Standorte fördern kann.

Was den weiteren Forschungsbedarf angeht, erscheint insbesondere die Entwicklung von Methoden zur Erfassung von Nachverdichtungspotenzialen in den bereits stark verdichteten Räumen ohne Flächenpotenziale gewinnbringend 
und lohnend. Ansätze wie Raum $+^{6}$ sind hierbei vielversprechend, die zur methodischen Weiterentwicklung genutzt werden können. So werden hier Innenentwicklungspotenziale kleinräumig unter anderem nach Parzellenstruktur, Bebauungsstand, Erschließung und Baureife erfasst. In der hier vorgestellten Methode wird zudem nicht zwischen Potenzialflächen für Gewerbe, Industrie oder Wohnen unterschieden. Mit dem Bedeutungsgewinn der urbanen Produktion in den letzten Jahren könnte eine differenziertere Betrachtung der Potenzialflächen nach unterschiedlichen Lagen und Standortqualitäten die Formulierung von passgenaueren Handlungsempfehlungen ermöglichen. In den USA gibt es bereits seit vielen Jahren umfangreiche Studien zu den sozialen Effekten von Metro-Stationen auf das direkte Umfeld (Kahn 2007; Padeiro/Louro/da Costa 2019). In Deutschland scheinen soziale Aspekte in bisherigen Analysen dahingegen unterrepräsentiert. Vorliegende Ergebnisse bieten aus der Sicht der Autoren daher einen guten Ausgangspunkt, um sich dieser Forschungslücke anzunähern, in dem kleinräumige demographische und sozioökonomische Daten in Beziehung gesetzt werden mit den erfassten TOD-Dimensionen.

Förderhinweis Deutsche Forschungsgemeinschaft (DI 1641/14-1 \& SI 932/10-1)

\section{Literatur}

Alfken, C.; Iwer, N. (2017): ruhrFIS-Flächeninformationssystem Ruhr. Monitoring Daseinsvorsorge 2017. Essen.

Anderhub, G.; Dorbritz, R.; Weidmann, U. (2008): Leistungsfähigkeitsbestimmung öffentlicher Verkehrssysteme. Zürich. = IVT-Schriftenreihe 139. https://doi.org/ 10.3929/ethz-b-000028520

Bahn.Ville 2-Konsortium (2010): Die Bahn als Rückgrat einer nachhaltigen Siedlungs- und Verkehrsentwicklung. Synthesebericht zum Projekt Bahn.Ville 2. München.

Banai, R. (1998): Transit-Oriented Development Suitability Analysis by the Analytic Hierarchy Process and a Geographic Information System: A Prototype Procedure. In: Journal of Public Transportation 2, 1, 43-65. https://doi. org/10.5038/2375-0901.2.1.3

Banai, R. (2005): Land Resource Sustainability for Urban Development: Spatial Decision Support System Prototype. In: Environmental Management 36, 2, 282-296. https://doi.org/10.1007/s00267-004-1047-0

Beckmann, G.; Dosch, F. (2018): Monitoring der Siedlungsflächenentwicklung. In: Behnisch, M.; Kretschmer, O.; Meinel, G. (Hrsg.): Flächeninanspruchnahme in

6 https://www.raumplus.ethz.ch/de/methodik/ (08.12.2020).
Deutschland. Auf dem Wege zu einem besseren Verständnis der Siedlungs- und Verkehrsflächenentwicklung. Berlin, 3-24. https://doi.org/10.1007/978-3-662-50305-8_1 Behnisch, M.; Kretschmer, O.; Meinel, G. (Hrsg.) (2018): Flächeninanspruchnahme in Deutschland. Auf dem Wege zu einem besseren Verständnis der Siedlungs- und Verkehrsflächenentwicklung. Berlin. https://doi.org/10. 1007/978-3-662-50305-8

Bertolini, L. (1996): Nodes and places: complexities of railway station redevelopment. In: European Planning Studies 4, 3, 331-345. https://doi.org/10.1080/ 09654319608720349

Bertolini, L. (1999): Spatial Development Patterns and Public Transport: The Application of an Analytical Model in the Netherlands. In: Planning Practice and Research 14, 2, 199-210. https://doi.org/10.1080/02697459915724

Boarnet, M. G.; Compin, N. S. (1999): Transit-Oriented Development in San Diego County. In: Journal of the American Planning Association 65, 1, 80-95. https://doi. org/10.1080/01944369908976035

Bose, M. (1994): Wirkungsanalyse eines stadtregionalen Siedlungsstrukturkonzeptes und Ansätze für eine Neuorientierung. Das Entwicklungsmodell für Hamburg und sein Umland. Hamburg. = Harburger Berichte zur Stadtplanung 4.

Büttner, B.; Kinigadner, J.; Ji, C.; Wright, B.; Wulfhorst, G. (2018): The TUM Accessibility Atlas: Visualizing Spatial and Socioeconomic Disparities in Accessibility to Support Regional Land-Use and Transport Planning. In: Networks and Spatial Economics 18, 2, 385-414. https:// doi.org/10.1007/s11067-017-9378-6

Calthorpe, P. (1993): The next American metropolis. Ecology, community, and the American Dream. New York.

Carlton, I. (2019): Transit Planners' Transit-Oriented Development-Related Practices and Theories. In: Journal of Planning Education and Research 39, 4, 508-519. https:// doi.org/10.1177/0739456X19878867

Cervero, R. (1984): Journal Report: Light Rail Transit and Urban Development. In: Journal of the American Planning Association 50, 2, 133-147. https://doi.org/10.1080/ 01944368408977170

Cervero, R. (1995): Sustainable new towns: Stockholm's rail-served satellites. In: Cities 12, 1, 41-51. https://doi. org/10.1016/0264-2751(95)91864-C

Cervero, R.; Arrington, G. B. (2008): Vehicle Trip Reduction Impacts of Transit-Oriented Housing. In: Journal of Public Transportation 11, 3, 1-17. https://doi.org/10. 5038/2375-0901.11.3.1

Cervero, R.; Kockelman, K. (1997): Travel demand and the 3Ds: Density, diversity, and design. In: Transportation Research Part D: Transport and Environment 2, 3, 199219. https://doi.org/10.1016/S1361-9209(97)00009-6 
Chen, X.; Lin, L. (2015): The node-place analysis on the "hubtropolis" urban form: The case of Shanghai Hongqiao air-rail hub. In: Habitat International 49, 445-453. https://doi.org/10.1016/j.habitatint.2015.06.013

Curtis, C. (2008): Evolution of the Transit-oriented Development Model for Low-density Cities: A Case Study of Perth's New Railway Corridor. In: Planning Practice and Research 23, 3, 285-302. https://doi.org/10.1080/ 02697450802423559

Dechent, J.; Wagner, I. (2020): Der Wohnungsmarkt aus Sicht der Bautätigkeitsstatistik. Entwicklung und Kennzahlen seit der Jahrtausendwende. In: Wirtschaft und Statistik: 72, 2, 46-60.

Dong, H. (2016): If You Build Rail Transit in Suburbs, Will Development Come? In: Journal of the American Planning Association 82, 4, 316-326. https://doi.org/10. 1080/01944363.2016.1215258

Fina, S. (2018): Ist die Konfiguration und Komposition der Flächennutzung messbar? In: Behnisch, M.; Kretschmer, O.; Meinel, G. (Hrsg.): Flächeninanspruchnahme in Deutschland. Auf dem Wege zu einem besseren Verständnis der Siedlungs- und Verkehrsflächenentwicklung. Berlin, 111-130. https://doi.org/10.1007/978-3662-50305-8_7

García-Palomares, J. C.; Gutiérrez, J.; Cardozo, O. D. (2013): Walking Accessibility to Public Transport: An Analysis Based on Microdata and GIS. In: Environment and Planning B: Planning and Design 40, 6, 1087-1102. https://doi.org/10.1068/b39008

Gilat, M.; Sussman, J. (2003): Coordinated Transportation and Land Use Planning in the Developing World: Case of Mexico City. In: Transportation Research Record 1859, 1, 102-109. https://doi.org/10.3141/1859-13

Greene, M.; Mora, R. I.; Figueroa, C.; Waintrub, N.; de Dios Ortúzar, J. (2017): Towards a sustainable city: Applying urban renewal incentives according to the social and urban characteristics of the area. In: Habitat International 68, 15-23. https://doi.org/10.1016/j.habitatint.2017. 03.004

Greene, M.; Mora, R.; Waintrub, N.; Figueroa, C.; de Dios Ortúzar, J. (2016): How do we densify and socially integrate our cities? On the efficiency of urban property incentives in the vicinity of mass transit stations. In: Revista de la construcción 15, 3, 77-86. https://doi.org/10. 4067/S0718-915X2016000300008

Hickman, R.; Hall, P. (2008): Moving the City East: Explorations into Contextual Public Transport-orientated Development. In: Planning Practice and Research 23, 3, 323339. https://doi.org/10.1080/02697450802423583

Higgins, C. D.; Kanaroglou, P. S. (2016): A latent class method for classifying and evaluating the performance of station area transit-oriented development in the Toronto region. In: Journal of Transport Geography 52, 61-72. https://doi.org/10.1016/j.jtrangeo.2016.02.012

Ibraeva, A.; Correia, G.; Silva, C.; Pais Antunes, A. (2020): Transit-oriented development: A review of research achievements and challenges. In: Transportation Research Part A: Policy and Practice 132, 110-130. https:// doi.org/10.1016/j.tra.2019.10.018

Jamme, H.-T.; Rodriguez, J.; Bahl, D.; Banerjee, T. (2019): A Twenty-Five-Year Biography of the TOD Concept: From Design to Policy, Planning, and Implementation. In: Journal of Planning Education and Research 39, 4, 409-428. https://doi.org/10.1177/0739456X19882073

Kahn, M. E. (2007): Gentrification Trends in New Transit-Oriented Communities: Evidence from 14 Cities That Expanded and Built Rail Transit Systems. In: Real Estate Economics 35, 2, 155-182. https://doi.org/10. 1111/j.1540-6229.2007.00186.x

Kamruzzaman, M.; Wood, L.; Hine, J.; Currie, G.; GilesCorti, B.; Turrell, G. (2014): Patterns of social capital associated with transit oriented development. In: Journal of Transport Geography 35, 144-155. https://doi.org/10. 1016/j.jtrangeo.2014.02.003

Kay, A. I.; Noland, R. B.; DiPetrillo, S. (2014): Residential property valuations near transit stations with transit-oriented development. In: Journal of Transport Geography 39, 131-140. https://doi.org/10.1016/j.jtrangeo.2014.06. 017

Kim, D.; Ahn, Y.; Choi, S.; Kim, K. (2016): Sustainable Mobility: Longitudinal Analysis of Built Environment on Transit Ridership. In: Sustainability 8, 10, 1016. https:// doi.org/10.3390/su8101016

Knowles, R. D. (2012): Transit Oriented Development in Copenhagen, Denmark: from the Finger Plan to Ørestad. In: Journal of Transport Geography 22, 251-261. https:// doi.org/10.1016/j.jtrangeo.2012.01.009

Kodinariya, T. M.; Makwana, P. R. (2013): Review on determining number of Cluster in K-Means Clustering. In: International Journal of Advance Research in Computer Science and Management Studies 1, 6, 90-95.

Liang, Y.; Du, M.; Wang, X.; Xu, X. (2020): Planning for urban life: A new approach of sustainable land use plan based on transit-oriented development. In: Evaluation and Program Planning 80, 101811. https://doi.org/10.1016/j. evalprogplan.2020.101811

Lin, J. J.; Gau, C. C. (2006): A TOD planning model to review the regulation of allowable development densities around subway stations. In: Land Use Policy 23, 3, 353360. https://doi.org/10.1016/j.landusepol.2004.11.003

Lin, J.-J.; Li, C.-N. (2008): A grey programming model for regional transit-oriented development planning. In: Papers in Regional Science 87, 1, 119-138. https://doi. org/10.1111/j.1435-5957.2007.00146.x 
Loo, B. P. Y.; Cheng, A. H. T.; Nichols, S. L. (2017): Transit-oriented development on greenfield versus infill sites: Some lessons from Hong Kong. In: Landscape and Urban Planning 167, 37-48. https://doi.org/10.1016/ j.landurbplan.2017.05.013

Lyu, G.; Bertolini, L.; Pfeffer, K. (2016): Developing a TOD typology for Beijing metro station areas. In: Journal of Transport Geography 55, 40-50. https://doi.org/10.1016/ j.jtrangeo.2016.07.002

Ma, X.; Chen, X.; Li, X.; Ding, C.; Wang, Y. (2018): Sustainable station-level planning: An integrated transport and land use design model for transit-oriented development. In: Journal of Cleaner Production 170, 1052-1063. https://doi.org/10.1016/j.jclepro.2017.09.182

Maretzke, S. (2014): Infrastrukturindikator 2012. Ein wichtiger Indikator für die Neuabgrenzung der Fördergebiete in Deutschland. Bonn. = BBSR-Analysen kompakt 05/2014.

Meyer, J. R.; Kain, J. F.; Wohl, M. (1965): The Urban Transportation Problem. Cambridge. https://doi.org/10.4159/ harvard.9780674421202

Monajem, S.; Nosratian, F. E. (2015): The evaluation of the spatial integration of station areas via the node place model; an application to subway station areas in Tehran. In: Transportation Research Part D: Transport and Environment 40, 14-27. https://doi.org/10.1016/j.trd.2015. 07.009

Münter, A.; Osterhage, F. (2018): Konzepte der Raumordnung. In: ARL - Akademie für Raumforschung und Landesplanung (Hrsg.): Handwörterbuch der Stadt- und Raumentwicklung. Hannover, 1183-1195.

National Academies of Sciences, Engineering, and Medicine (2004): Transit-Oriented Development in the United States: Experiences, Challenges, and Prospects. Washington, D.C. $=$ TCRP Report 102. https://doi.org/10.17226/ 23360

Niu, S.; Hu, A.; Shen, Z.; Lau, S. S. Y.; Gan, X. (2019): Study on land use characteristics of rail transit TOD sites in new towns - taking Singapore as an example. In: Journal of Asian Architecture and Building Engineering 18, 1, 16-27. https://doi.org/10.1080/13467581.2019.1586712

Ogra, A.; Ndebele, R. (2014): The role of 6Ds: density, diversity, design, destination, distance, and demand management in transit oriented development (TOD). In: Proceedings of the NICHE-2014 Neo-International Conference on Habitable Environments. Jalandhar, 539-546.

Osterhage, F. (2018): The End of Reurbanisation? Phases of Concentration and Deconcentration in Migratory Movements in North Rhine-Westphalia. In: Comparative Population Studies 43, 131-156. https://doi.org/10.12765/ CPoS-2018-10

Padeiro, M.; Louro, A.; da Costa, N. M. (2019): Transit- oriented development and gentrification: a systematic review. In: Transport Reviews 39, 6, 733-754. https://doi. org/10.1080/01441647.2019.1649316

Papa, E.; Bertolini, L. (2015): Accessibility and TransitOriented Development in European metropolitan areas. In: Journal of Transport Geography 47, 70-83. https:// doi.org/10.1016/j.jtrangeo.2015.07.003

Paulsen, K. (2014): The Effects of Land Development on Municipal Finance. In: Journal of Planning Literature 29, 1, 20-40. https://doi.org/10.1177/0885412213497982

Paulsson, A. (2020): The city that the metro system built: Urban transformations and modalities of integrated planning in Stockholm. In: Urban Studies 57, 14, 2936-2955. https://doi.org/10.1177/0042098019895231

Perry, C. A. (1929): City Planning for Neighborhood Life. In: Social Forces 8, 1, 98-100. https://doi.org/10.2307/ 2570059

Pitot, M.; Yigitcanlar, T.; Sipe, N.; Evans, R. (2006): Land use and public transport accessibility index (LUPTAI) tool: the development and pilot application of LUPTAI for the Gold Coast. In: Grainger, C. (Hrsg.): Proceedings of the 29th Australian Transport Research Forum. Crawley, 1-18.

Renne, J. L.; Tolford, T.; Hamidi, S.; Ewing, R. (2016): The Cost and Affordability Paradox of Transit-Oriented Development: A Comparison of Housing and Transportation Costs Across Transit-Oriented Development, Hybrid and Transit-Adjacent Development Station Typologies. In: Housing Policy Debate 26, 4-5, 819-834. https://doi. org/10.1080/10511482.2016.1193038

Reusser, D. E.; Loukopoulos, P.; Stauffacher, M.; Scholz, R. W. (2008): Classifying railway stations for sustainable transitions - balancing node and place functions. In: Journal of Transport Geography 16, 3, 191-202. https:// doi.org/10.1016/j.jtrangeo.2007.05.004

Sahu, A. (2018): A methodology to modify land uses in a transit oriented development scenario. In: Journal of Environmental Management 213, 467-477. https://doi. org/10.1016/j.jenvman.2017.12.004

Schmitt, M.; Seidl, I. (2006): Der Einfluss des Bodenpreisniveaus auf die Bebauungsdichte von Wohnareal. In: Raumforschung und Raumordnung 64, 2, 93-103. https:// doi.org/10.1007/BF03183167

Seidemann, D. (2016): Wechselwirkungen von Siedlungsentwicklung und Schienenverkehr. Bedeutung von Siedlungsflächenreserven für den Schienenpersonenverkehr unter besonderer Berücksichtigung der Innenentwicklung am Beispiel der Regionen Mittlerer Oberrhein, Rhein-Neckar und Ostwürttemberg. Zürich.

Shannon, C. E.; Weaver, W. (1964): The mathematical theory of communication. Urbana.

Singh, Y.J.; Fard, P.; Zuidgeest, M.; Brussel, M.; van Maar- 
seveen, M. (2014): Measuring transit oriented development: a spatial multi criteria assessment approach for the City Region Arnhem and Nijmegen. In: Journal of Transport Geography 35, 130-143. https://doi.org/10.1016/j. jtrangeo.2014.01.014

Spars, G. (2017): Bodenpreise und Immobilienmärkte als Verstärker sozialräumlicher Polarisierung. In: HeinrichBöll-Stiftung (Hrsg.): Geteilte Räume. Strategien für mehr sozialen und räumlichen Zusammenhalt. Berlin, 79-95. = Schriften zu Wirtschaft und Soziales 21.

Strong, K. C.; Ozbek, M. E.; Sharma, A.; Akalp, D. (2017): Decision Support Framework for Transit-Oriented Development Projects. In: Transportation Research Record 2671, 1, 51-58. https://doi.org/10.3141/2671-06

Thomas, R.; Pojani, D.; Lenferink, S.; Bertolini, L.; Stead, D.; van der Krabben, E. (2018): Is transit-oriented development (TOD) an internationally transferable policy concept? In: Regional Studies 52, 9, 1201-1213. https:// doi.org/10.1080/00343404.2018.1428740

Tumlin, J.; Millard-Ball, A. (2006): Parking for TransitOriented Development. In: ITE 2006 Annual Meeting and Exhibit Compendium of Technical Papers: August 6-9, 2006. Milwaukee.

Xu, T.; Zhang, M.; Aditjandra, P. T. (2016): The impact of urban rail transit on commercial property value: New evidence from Wuhan, China. In: Transportation Research Part A: Policy and Practice 91, 223-235. https://doi.org/ 10.1016/j.tra.2016.06.026

Yang, K.; Pojani, D. (2017): A Decade of Transit Oriented Development Policies in Brisbane, Australia: Development and Land-Use Impacts. In: Urban Policy and Research 35, 3, 347-362. https://doi.org/10.1080/08111146. 2017.1294537

Yu, H.; Pang, H.; Zhang, M. (2018): Value-added effects of transit-oriented development: The impact of urban rail on commercial property values with consideration of spatial heterogeneity. In: Papers in Regional Science 97, 4, 1375-1396. https://doi.org/10.1111/pirs. 12304

Zemp, S.; Stauffacher, M.; Lang, D. J.; Scholz, R. W. (2011): Classifying railway stations for strategic transport and land use planning: Context matters! In: Journal of Transport Geography 19, 4, 670-679. https://doi.org/ 10.1016/j.jtrangeo.2010.08.008

Zhao, P.; Yang, H.; Kong, L.; Liu, Y.; Liu, D. (2018): Disintegration of metro and land development in transition China: A dynamic analysis in Beijing. In: Transportation Research Part A: Policy and Practice 116, 290-307. https://doi.org/10.1016/j.tra.2018.06.017 